TITLE:

\title{
Regeneration cycle and the covariant Lyapunov vectors in a minimal wall turbulence.
}

\section{$\operatorname{AUTHOR}(\mathrm{S})$ :}

Inubushi, Masanobu; Takehiro, Shin-Ichi; Yamada, Michio

\section{CITATION:}

Inubushi, Masanobu ...[et al]. Regeneration cycle and the covariant Lyapunov vectors in a minimal wall turbulence.. Physical review E 2015, 92(2): 023022.

ISSUE DATE:

2015-08-24

URL:

http://hdl.handle.net/2433/201954

RIGHT:

(C)2015 American Physical Society 
PHYSICAL REVIEW E 92, 023022 (2015)

\title{
Regeneration cycle and the covariant Lyapunov vectors in a minimal wall turbulence
}

\author{
Masanobu Inubushi, ${ }^{*}$ Shin-ichi Takehiro, ${ }^{\dagger}$ and Michio Yamada ${ }^{\ddagger}$ \\ Research Institute for Mathematical Sciences, Kyoto University, Kyoto, Japan \\ (Received 21 January 2013; revised manuscript received 9 June 2015; published 24 August 2015)
}

\begin{abstract}
Considering a wall turbulence as a chaotic dynamical system, we study regeneration cycles in a minimal wall turbulence from the viewpoint of orbital instability by employing the covariant Lyapunov analysis developed by F. Ginelli et al. [Phys. Rev. Lett. 99, 130601 (2007)]. We divide the regeneration cycle into two phases and characterize them with the local Lyapunov exponents and the covariant Lyapunov vectors of the Navier-Stokes turbulence. In particular, we show numerically that phase (i) is dominated by instabilities related to the sinuous mode and the streamwise vorticity, and there is no instability in phase (ii). Furthermore, we discuss a mechanism of the regeneration cycle, making use of an energy budget analysis.
\end{abstract}

DOI: 10.1103/PhysRevE.92.023022

PACS number(s): 47.52.+j, 05.45.-a, 47.27.N-

\section{INTRODUCTION}

Toward an understanding of wall turbulence based on the Navier-Stokes equations, we characterize a wall turbulence in terms of orbital instability of chaos. The orbital instability is quantified by Lyapunov exponents and Lyapunov vectors. While the Lyapunov exponent $\lambda$ is an exponential growth rate of the norm of the perturbation vector added to a chaotic orbit, the Lyapunov vector $\boldsymbol{y}$ is the perturbation vector whose norm grows exponentially as $\|\boldsymbol{y}(t)\| \propto e^{\lambda t}$. The Lyapunov exponents and vectors characterize stabilities of a chaotic attractor, just as eigenvalues and eigenvectors characterize stabilities of a fixed point attractor. Ginelli et al. [1] developed the covariant Lyapunov analysis, proposing a numerical algorithm to calculate both Lyapunov exponents and vectors. While the covariant Lyapunov analysis has been applied to various dynamical systems to study their chaotic properties such as hyperbolicity, effective dimension, and inertial manifold [2-7] (see review papers [8-10] and references therein), it has not been applied to the three-dimensional Navier-Stokes equations. In this paper, by using this algorithm, we study the orbital instability of the regeneration cycle in the wall turbulence governed by the Navier-Stokes equations.

Wall turbulence has been studied as a typical turbulence associated with the wall boundary. To find out a "minimal" mechanism producing wall turbulence, Jimenez and Moin [11] and Hamilton et al. [12] searched numerically the minimal size of the periodic box (minimal flow unit) in which we can observe turbulence. As a result, in the minimal flow units, they found regeneration cycles consisting of breakdown and reformation of the coherent structures such as streamwise vortices and streaks which are high or low streamwise velocity regions in Poiseuille turbulence [11] and in Couette turbulence [12]. The regeneration cycle has been observed in many types of wall turbulence (Panton [13]) and was recently observed in experiments of boundary layer turbulence by Duriez et al. [14].

In order to describe the regeneration cycle, Hamilton et al. [12] and Waleffe [15] proposed a mechanism (which they call

\footnotetext{
*inubushi.masanobu@ lab.ntt.co.jp; Present address: NTT Communication Science Laboratories, NTT Corporation.

${ }^{\dagger}$ takepiro@gfd-dennou.org

${ }^{\ddagger}$ yamada@kurims.kyoto-u.ac.jp
}

self-sustaining process) which consists of streak instability, regeneration of the streamwise vortices, and reformation of the streaks, by modeling the streaks and the streamwise vortices. On the streak instability, Schoppa and Hussain [16] investigated linear stability of model streaks numerically and found that these models are linearly unstable to the sinuous instability mode (see Fig. 9 in [16]) which causes meandering of the straight streak as observed by Hamilton et al. [12]. Linear stability of a corrugated vortex sheet, which is an inviscid model of the streak, was studied by Kawahara et al. [17]. They found the vortex sheet is linearly unstable to the sinuous disturbance in a long-wave limit. There are numerous studies on linear stability of model streaks including the above models (see [17] and references therein) and most of them suggest that the sinuous mode is the most unstable perturbation.

Following the meanderings of the straight streaks, the flow changes into fully three-dimensional turbulence, and streamwise vortices are expected to be generated. To understand this process, many mechanisms have been proposed such as Waleffe [15] and Jiménez and Moin [11] (see [18]). Once the streamwise vortices are generated, these vortices advect the gradient of the streamwise velocity in the cross-streamwise plane, which forms the streak structures. Kawahara [18] showed that an analytical model of the streamwise vortex forms the streak structures by the above mechanism. Waleffe [15] derived a low-dimensional ODE model for understanding of the regeneration cycle. While these models and their results are suggestive, the mechanisms of the regeneration cycle should be clarified based on the full Navier-Stokes equations.

One of the crucial steps toward understanding of the regeneration cycle on the basis of the full Navier-Stokes equation is finding of the unstable periodic orbit (UPO) by Kawahara and Kida [19] which approximates turbulent statistics very well. Recently, a lot of invariant solutions of the full Navier-Stokes equation and the (homoclinic and heteroclinic) connections between them have been found numerically and used to clarify the state space structures for understanding mechanisms of the regeneration cycle [20-24] (see Kawahara [25] for the detailed review).

The main questions we study here are as follows: Can we clarify the mechanisms underlying the regeneration cycle without using the ad hoc models? How does the orbital instabilities play roles in the regeneration cycle? To answer these questions, we characterize the regeneration cycle in the minimal Couette 
turbulence with the covariant Lyapunov analysis applied to the full Navier-Stokes equation. We formulate the problem in Sec. II, and describe the covariant Lyapunov analysis and the numerical method in Sec. III. We show the turbulent behaviors of the minimal Couette turbulence, particular the regeneration cycle in Sec. IV. In Sec. V, we show the main result of this paper: the orbital instability of the regeneration cycle in the minimal Couette turbulence. In order to discuss the streak reformation, we study the energy budget of the minimal Couette turbulence in Sec. VI. Finally, we give our conclusion and discussion in Sec. VII.

\section{MINIMAL COUETTE FLOW SYSTEM}

Plane Couette flow is a fluid system where incompressible viscous fluid is in between upper and lower walls and the fluid motion is driven by the walls moving in the opposite direction as shown in Fig. 1. We set the streamwise flux and the spanwise mean pressure gradient to be zero.

The nondimensionalized Navier-Stokes equation and the incompressible condition is

$$
\begin{aligned}
\frac{\partial \boldsymbol{u}}{\partial t}+(\boldsymbol{u} \cdot \nabla) \boldsymbol{u} & =-\nabla p+\frac{1}{\operatorname{Re}} \nabla^{2} \boldsymbol{u}, \\
\nabla \cdot \boldsymbol{u} & =0,
\end{aligned}
$$

where $\boldsymbol{u}=\left(u_{x}, u_{y}, u_{z}\right)$ is the velocity, and $p$ is the pressure defined in the domain $(x, y, z) \in\left[0, L_{x}\right] \times\left[0, L_{y}\right] \times[-1,1]$. We use the nonslip boundary condition on the walls $(z= \pm 1)$ :

$$
\begin{gathered}
u_{x}(x, y, \pm 1)= \pm 1, \\
u_{z}(x, y, \pm 1)=u_{y}(x, y, \pm 1)=0,
\end{gathered}
$$

and the periodic boundary condition in a horizontal direction:

$$
\begin{gathered}
\boldsymbol{u}(x, y, z)=\boldsymbol{u}\left(x+L_{x}, y, z\right)=\boldsymbol{u}\left(x, y+L_{y}, z\right), \\
\nabla p(x, y, z)=\nabla p\left(x+L_{x}, y, z\right)=\nabla p\left(x, y+L_{y}, z\right) .
\end{gathered}
$$

The Reynolds number is set to be $\mathrm{Re}=400$ and the domain size is the minimal flow unit size: $L_{x}=1.755 \pi, L_{y}=1.2 \pi$. Decomposing the flow field into the toroidal and poloidal potentials, we compute the time evolution of the potentials.

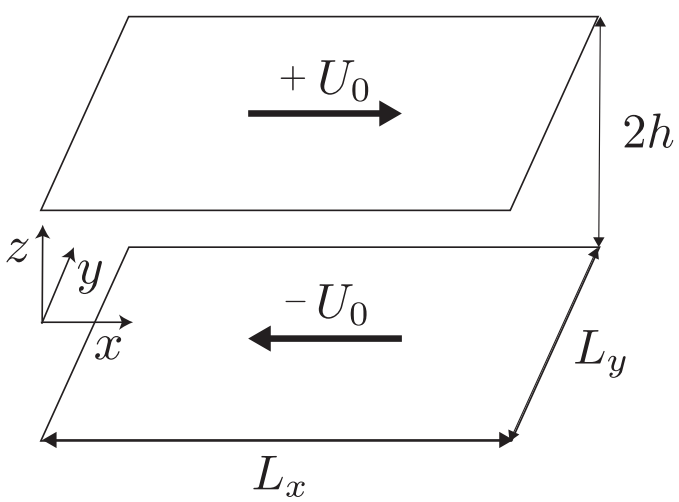

FIG. 1. Illustration of the plane Couette flow system. $x, y, z$ directions are referred to as streamwise, spanwise, wall-normal directions, respectively.
The de-aliased Fourier expansions (2/3 rule) are employed in the horizontal $(x-y)$ directions, and the Chebyshev tau methods are employed in the wall-normal $(z)$ direction: $\psi(x, y, z)=$ $\sum_{k=-K M}^{K M} \sum_{l=-L M}^{L M} \sum_{m=0}^{M M} \hat{\psi}_{(k, l, m)} e^{i(\alpha k x+\beta l y)} T_{m}(z), \quad$ where $\hat{\psi}_{(k, l, m)}$ is the expansion coefficient, $\alpha=2 \pi / L_{x}$ and $\beta=2 \pi / L_{y}$ are the fundamental streamwise and spanwise wave numbers, respectively, and $T_{m}(z)$ is the $m$ th-order Chebyshev polynomial. We set the truncation mode numbers $K M=8$ ( $x$ direction), $L M=8$ ( $y$ direction), $M M=32(z$ direction), and the computational grid points are $32 \times 32 \times 33$ ( $x, y, z$ directions). The dimension of the dynamical system $N \quad$ is $\quad N=2[(2 K M+1)(2 L M+1)-1](M M+1)+$ $2(M M+1)=19074$. The time integration is performed with the second-order Adams-Bashforth method with a time step width $\Delta t=1.0 \times 10^{-3}$. The CFL number is less than 0.1 which is less than Philip and Manneville [26] use in the similar setting. The friction Reynolds number $\operatorname{Re}_{\tau}\left(=u_{\tau} h / v\right)$ is $\operatorname{Re}_{\tau}=34.0$ and the periods of the domain in the streamwise and spanwise directions normalized by $l_{\tau}=v / u_{\tau}$ are $L_{x}^{+}=L_{x} / l_{\tau}=187$ and $L_{y}^{+}=L_{y} / l_{\tau}=128$, respectively, which is in good agreement with the values reported in Kawahara [19]. The grid spacing in the $x, y$, and $z$ directions normalized by $l_{\tau}$ is $\Delta x^{+}=5.9, \Delta y^{+}=4.0$, and $\Delta z^{+}=0.16-3.3$ (the minimum-maximum grid spacing), which is comparable to those in most direct numerical simulations [12]. We used the library for spectral transform ISPACK [27], its Fortran90 wrapper library the SPMODEL library [28], and the subroutine of LAPACK. For drawing the figures, the products of the Dennou Ruby project [29] and gnuplot were used.

\section{COVARIANT LYAPUNOV ANALYSIS}

In this section we describe in some detail the computational method for covariant Lyapunov analysis proposed by Ginelli et al. (2007) [1], and for its application to the present NavierStokes wall turbulence.

Let us consider a discrete dynamical system $\boldsymbol{f}: \mathbb{R}^{N} \rightarrow$ $\mathbb{R}^{N}$, and we write time evolution of a state point $\boldsymbol{x}_{n} \in \mathbb{R}^{N}$ as $\boldsymbol{x}_{n+1}=\boldsymbol{f}\left(\boldsymbol{x}_{n}\right)$. Infinitesimal perturbation vectors $\boldsymbol{y}_{n}^{(j)}(j=$ $1,2, \ldots, N)$ added to a state point $\boldsymbol{x}_{n}$ on the orbit evolve as $\boldsymbol{y}_{n+1}^{(j)}=D \boldsymbol{f}_{n} \boldsymbol{y}_{n}^{(j)}$ where $D \boldsymbol{f}_{n}$ is a $N \times N$ Jacobian matrix at $\boldsymbol{x}_{n}$. Then, the $j$ th Lyapunov exponent $\lambda_{j}\left(\lambda_{1} \geqslant \lambda_{2} \geqslant \cdots \geqslant\right.$ $\left.\lambda_{N}\right)$ and its corresponding covariant Lyapunov vector $\boldsymbol{y}_{0}^{(j)}$ at a state point $\boldsymbol{x}_{0}$ are defined as follows:

$$
\lambda_{j}=\lim _{k \rightarrow \pm \infty} \frac{1}{k} \ln \left\|D \boldsymbol{f}^{k} \boldsymbol{y}_{0}^{(j)}\right\|,
$$

where $D \boldsymbol{f}^{k}=D \boldsymbol{f}_{k-1} \circ D \boldsymbol{f}_{k-2} \circ \cdots \circ D \boldsymbol{f}_{0}$. A set of Lyapunov exponents $\left\{\lambda_{1}, \lambda_{2}, \cdots, \lambda_{N}\right\}$ is referred to as a Lyapunov spectrum.

The definition of the Lyapunov exponents and the covariant Lyapunov vectors (6) does not depend on the choice of the norm. The state space is finite dimensional and thus all norms are equivalent; i.e., for arbitrary norms $\|\cdot\|_{A},\|\cdot\|_{B}$, there exist positive real numbers $\alpha_{i}(i=1,2)$ such that $\alpha_{1}\|x\|_{A} \leqslant\|x\|_{B} \leqslant \alpha_{2}\|x\|_{A}$ for all $x \in \mathbb{R}^{N}$. If we consider $\left\|D \boldsymbol{f}^{k} \boldsymbol{y}_{0}^{(j)}\right\|_{A}$ and $\left\|D \boldsymbol{f}^{k} \boldsymbol{y}_{0}^{(j)}\right\|_{B}$ in the definition (6), there exist $\alpha_{1}$ and $\alpha_{2}$ such that $\alpha_{1}\left\|D \boldsymbol{f}^{k} \boldsymbol{y}_{0}^{(j)}\right\|_{A} \leqslant\left\|D \boldsymbol{f}^{k} \boldsymbol{y}_{0}^{(j)}\right\|_{B} \leqslant$ 
$\alpha_{2}\left\|D \boldsymbol{f}^{k} \boldsymbol{y}_{0}^{(j)}\right\|_{A}$. Clearly, if $1 / k \ln \left(\left\|D \boldsymbol{f}^{k} \boldsymbol{y}_{0}^{(j)}\right\|_{A}\right) \rightarrow \lambda_{j}(k \rightarrow$ $\infty)$, then $1 / k \ln \left(\alpha_{i}\left\|D \boldsymbol{f}^{k} \boldsymbol{y}_{0}^{(j)}\right\|_{A}\right) \rightarrow \lambda_{j}(k \rightarrow \infty)$ for $i=1,2$. Therefore, we obtain $1 / k \ln \left(\left\|D \boldsymbol{f}^{k} \boldsymbol{y}_{0}^{(j)}\right\|_{B}\right) \rightarrow \lambda_{j}(k \rightarrow \infty)$ from the above inequality, implying the definition (6) does not depend on the choice of the norm. Moreover, an infinitesimal perturbation vector that is linearly dependent on $\boldsymbol{y}_{0}^{(j)}$ (i.e., $\left.a \boldsymbol{y}_{0}^{(j)}, a \in \mathbb{R}\right)$ gives the same Lyapunov exponent $\lambda_{j}$. Thus, the important thing is not the norm of the covariant Lyapunov vector, but the direction of it. See [8-10] for more detailed descriptions and a mathematically rigorous definition of the Lyapunov exponents and vectors (sometimes referred to as Oseledets' splitting). The covariant Lyapunov vector indicates the intrinsic (i.e., norm-independent) stable/unstable direction along the chaotic orbit, which is expected to indicate instability mechanisms of turbulence.

The numerical algorithm of covariant Lyapunov analysis developed by Ginelli et al. (2007) gives both the Lyapunov exponents $\lambda_{j}$ and the corresponding Lyapunov vectors $\boldsymbol{y}_{n}^{(j)}$ at a state point $\boldsymbol{x}_{n}$ on the attractor. The Lyapunov vectors are not orthogonal to each other in general. However, before the algorithm was developed, there had been no efficient algorithm to calculate the Lyapunov vectors, and instead we just could obtain orthogonalized Lyapunov vectors which depend on the norm we use [30]. The covariant Lyapunov analysis paved the way to calculate the Lyapunov vectors as defined above. While Ginelli et al. refer to these Lyapunov vectors as covariant Lyapunov vectors, we simply refer to them as Lyapunov vectors or Lyapunov modes.

Here, we briefly review the numerical algorithm of covariant Lyapunov analysis [1,9]. Let us consider an orthogonal matrix $Q_{0}$ whose column vectors are arbitrary perturbation vectors orthogonal to each other $\boldsymbol{q}_{0}^{i}(i=1,2, \ldots, N)$ as $Q_{0}=$ $\left(\boldsymbol{q}_{0}^{1}\left|\boldsymbol{q}_{0}^{2}\right| \cdots \mid \boldsymbol{q}_{0}^{N}\right)$. The matrix evolves as $\tilde{Q}_{i+1}=D \boldsymbol{f}_{i} Q_{i}$. In general, $\tilde{Q}_{i}$ is not an orthogonal matrix, and we perform the Gram-Schmidt decomposition as $\tilde{Q}_{i+1}=Q_{i+1} R_{i+1}$ where $Q_{i+1}$ is an orthogonal matrix and $R_{i}$ is an upper-triangular matrix. For later use, the matrices $Q_{i}, R_{i}$ have to be stored. Continuing this procedure, i.e., transferring the orthogonal matrix $Q_{i}$ by the Jacobian matrix $D f_{i}$ and performing the Gram-Schmidt decomposition of $\tilde{Q}_{i}$ for sufficient times (we write $n \gg 0$ ), the first column vector $\boldsymbol{q}_{n}^{1}$ of $Q_{n}$ converges to a basis vector that spans an unstable subspace $V_{n}^{1}$ spanned by the Lyapunov vector $\boldsymbol{y}_{n}^{1}$, the column vectors $\boldsymbol{q}_{n}^{1}$ and $\boldsymbol{q}_{n}^{2}$ converge to orthogonal basis vectors that span an unstable subspace $V_{n}^{2}$ spanned by the Lyapunov vectors $\boldsymbol{y}_{n}^{1}$ and $\boldsymbol{y}_{n}^{2}$, and so on; the column vectors $\boldsymbol{q}_{n}^{1}, \boldsymbol{q}_{n}^{2}, \ldots, \boldsymbol{q}_{n}^{j}(j=1,2, \ldots, N)$ are the orthogonal basis vectors that span an unstable subspace $V_{n}^{j}$ spanned by the Lyapunov vectors $\boldsymbol{y}_{n}^{1}, \boldsymbol{y}_{n}^{2}, \ldots, \boldsymbol{y}_{n}^{j}$. Let $\boldsymbol{v}_{l}^{j} \in V_{l}^{j} \backslash V_{l}^{j-1}$ be an arbitrary vector, where $l \gg n$. When the vector $\boldsymbol{v}_{l}^{j}$ is transferred backward in time sufficiently, $\boldsymbol{v}_{l}^{j}$ converges to the $j$ th Lyapunov vector $\boldsymbol{y}_{l}^{j}$, and we obtain $\boldsymbol{v}_{m}^{j}$ as an approximation of $\boldsymbol{y}_{m}^{j}$ where $n \leqslant m \ll l$. This is because a direction along $\boldsymbol{y}_{m}^{j}$ is the most unstable direction in the backward time dynamics in $V_{m}^{j}$ (i.e., the most stable direction in the forward time dynamics in $V_{m}^{j}$ ). To calculate $\boldsymbol{v}_{m}^{j}$, let $U_{l}=\left(\boldsymbol{v}_{l}^{1}\left|\boldsymbol{v}_{l}^{2}\right| \cdots \mid \boldsymbol{v}_{l}^{N}\right)$ and $C_{l}$ be an upper-triangular matrix such that $U_{l}=Q_{l} C_{l}$. Substituting this into $U_{l+1}=$
$D f_{l} U_{l}$ and performing the Gram-Schmidt decomposition, we obtain $C_{l+1}=R_{l+1} C_{l}$; i.e., the coefficient matrix $C_{l}$ evolves backward in time as $C_{l-1}=R_{l}^{-1} C_{l}$. Once we obtain the matrix $C_{m}$, the vector $\boldsymbol{v}_{m}^{j}$ can be calculated as the $j$ th column vector of $U_{m}=Q_{m} C_{m}$. By using this algorithm, we obtain the vector $\boldsymbol{v}_{i}^{j}(j=1,2, \ldots, N)$ for $n<i<m$ which approximates the $j$ th Lyapunov vector $\boldsymbol{y}_{i}^{j}$, while discarding the data such as $Q_{i}$ and $U_{i}$ for $0 \leqslant i \leqslant n$ and $m \leqslant i \leqslant l$ as the transient data.

As for the numerical calculation of the minimal Couette flow, it is difficult computationally to calculate all of the Lyapunov vectors $\boldsymbol{y}^{(j)}(t)(j=1,2, \ldots, N)$ since the dimension of the dynamical system we study here is too high $(N=19074)$. Thus, we focused our attention on the first 30 Lyapunov vectors, i.e., $\boldsymbol{y}^{(j)}(t)(j=1,2, \ldots, M ; M=30)$. We calculated the time evolution of the $j$ th perturbed flow $(j=1,2, \ldots, M)$ governed by the linearized Navier-Stokes equation:

$$
\begin{aligned}
\frac{\partial \boldsymbol{u}^{j}}{\partial t}+\left(\boldsymbol{u}^{j} \cdot \nabla\right) \boldsymbol{u}+(\boldsymbol{u} \cdot \nabla) \boldsymbol{u}^{j} & =-\nabla p^{j}+\frac{1}{\operatorname{Re}} \nabla^{2} \boldsymbol{u}^{j}, \\
\nabla \cdot \boldsymbol{u}^{j} & =0,
\end{aligned}
$$

while calculating the base flow $\boldsymbol{u}$ governed by the NavierStokes equations (1) simultaneously, where $\boldsymbol{u}^{j}=\left(u_{x}^{j}, u_{y}^{j}, u_{z}^{j}\right)$ and $p^{j}$ are the velocity and pressure field of the perturbed flow. The boundary conditions of the perturbed flows are the same as those of the base flow except that of the streamwise flow on the walls: $u_{x}^{j}(x, y, \pm 1)=0$. Here, we considered a perturbation vector $\boldsymbol{q}^{j}(t) \in \mathbb{R}^{N}$ that is a vector whose components are the expansion coefficients of the perturbed flow field $\boldsymbol{u}^{j}(t)$; i.e., the vector $\boldsymbol{q}^{j}(t)$ determines the flow field $\boldsymbol{u}^{j}(t)$ and vice versa. The initial conditions of the perturbed flows $\boldsymbol{u}^{j}(0)(j=1,2, \ldots, M)$ are arbitrary as long as they are satisfying the boundary conditions and orthogonal to each other. The initial conditions of the perturbed flows $\boldsymbol{u}^{j}(0)(j=1,2, \ldots, M)$ correspond to $Q_{0}=\left(\boldsymbol{q}_{0}^{1}\left|\boldsymbol{q}_{0}^{2}\right| \cdots \mid \boldsymbol{q}_{0}^{M}\right)$. We calculated the time evolution of the perturbed flow $\boldsymbol{u}^{j}$ by solving the Eq. (7), and performed the Gram-Schmidt decomposition at each $T=1$, which correspond to $\tilde{Q}_{i+1}=D \boldsymbol{f}_{i} Q_{i}$ and $\tilde{Q}_{i+1}=Q_{i+1} R_{i+1}$ in the above algorithm. In our case, $Q_{i}$ is a $N \times M$ matrix and $R_{i}$ is a $M \times M$ matrix. The inner product we use here is defined by $\left(\boldsymbol{y}_{1}, \boldsymbol{y}_{2}\right)_{\mathcal{E}}=\frac{1}{2}\left\langle\boldsymbol{\omega}_{1} \cdot \boldsymbol{\omega}_{2}\right\rangle_{V}$ where $\omega_{i}(i=1,2)$ is the vorticity vector field determined by the perturbation vector $\boldsymbol{y}_{i}$ and $\langle\cdot\rangle_{V}$ denotes the volume average: $\langle\cdot\rangle_{V}=\frac{1}{2 L_{x} L_{y}} \int_{z=-1}^{z=+1} \int_{y=0}^{y=L_{y}} \int_{x=0}^{x=L_{x}} \cdot d x d y d z$. We used an induced norm from the inner product $(\cdot, \cdot)_{\mathcal{E}}$ which is a volume average of enstrophy: $\|\boldsymbol{y}\|^{2}=(\boldsymbol{y}, \boldsymbol{y})_{\mathcal{E}}$. By using the stored matrices $Q_{i}, R_{i}$, we obtained the Lyapunov vectors from the backward time evolution $C_{i-1}=R_{i}^{-1} C_{i}$ and $U_{i}=Q_{i} C_{i}$ as described above.

We obtained the Lyapunov vectors for $T=23000(=m-$ $n-1)$ while discarding the transient data for $T=5000$ (= $n+1=m-l+1)$. The Lyapunov exponents are calculated from both the Lyapunov vectors and the conventional method using Gram-Schmidt decomposition [30], and we confirmed that these values of the Lyapunov exponents calculated by different methods show good agreement. 


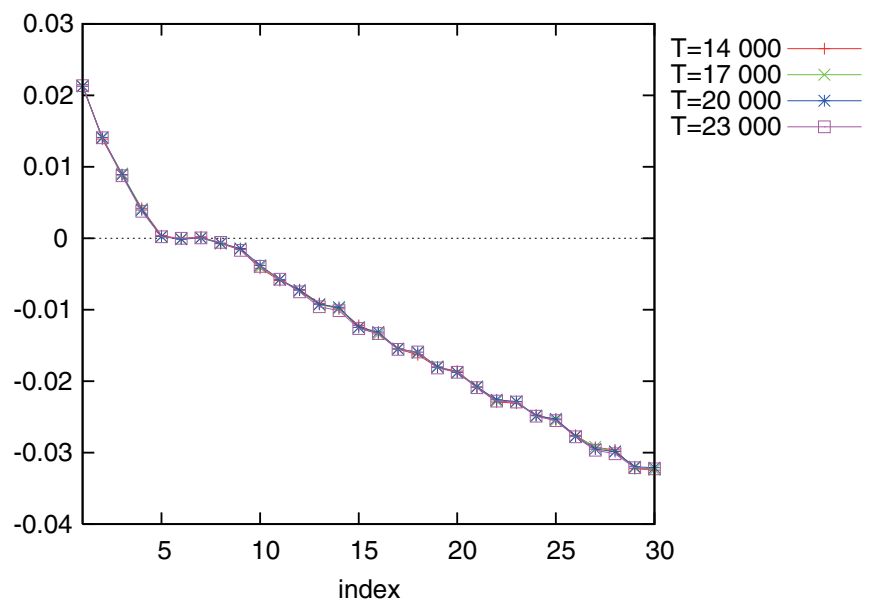

FIG. 2. (Color online) Lyapunov spectrum with the different averaging time $T$.

\section{A. Check for numerical precision}

We also confirmed that the qualitative properties of the results do not depend on the average time and other free parameters in the calculation. Here we show a numerical convergence of the Lyapunov exponents by varying the average time as $T=14000$ (the red line), 17000 (the green line), 20000 (the blue line), and 23000 (the pink line) in Fig. 2. Figure 2 shows the leading 30 Lyapunov exponents with the different averaging time. We see that the Lyapunov exponents seem to be independent of the averaging time. Figure 3 shows a closeup of the Fig. 2 focusing on the zero Lyapunov exponents (5th-8th Lyapunov exponents). Although the exponents do not seem to converge uniformly, the longer the averaging time is, the closer to zero the 5th-7th Lyapunov exponents are. We concluded that the 5th-7th Lyapunov exponents are zero Lyapunov exponents, and thus the number of the positive Lyapunov exponents is four (i.e., 1st-4th Lyapunov exponents).

We employ the de-aliased (2/3 rule) Fourier expansions in the streamwise $(x)$ and spanwise $(y)$ directions, and

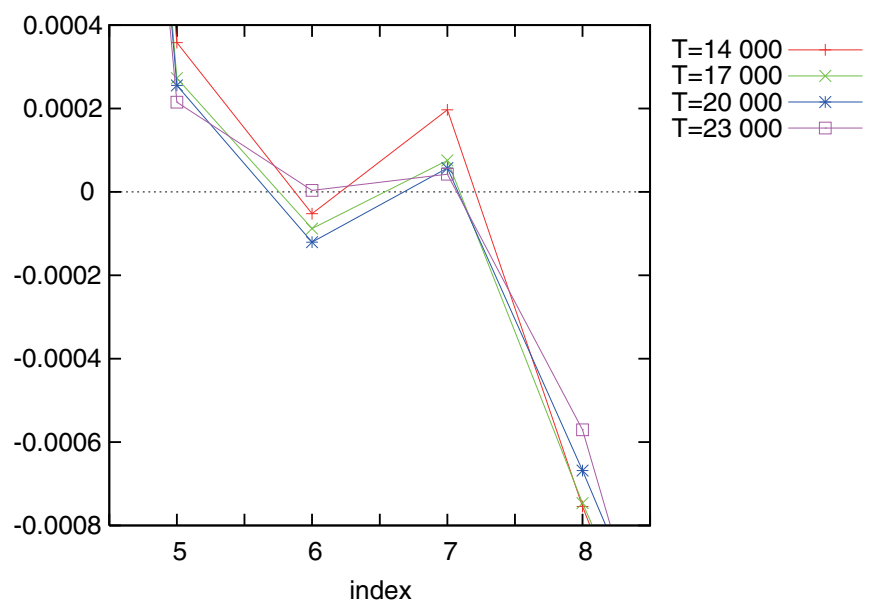

FIG. 3. (Color online) Closeup of the Fig. 2 focusing on the zero Lyapunov exponents (5th-8th Lyapunov exponents).

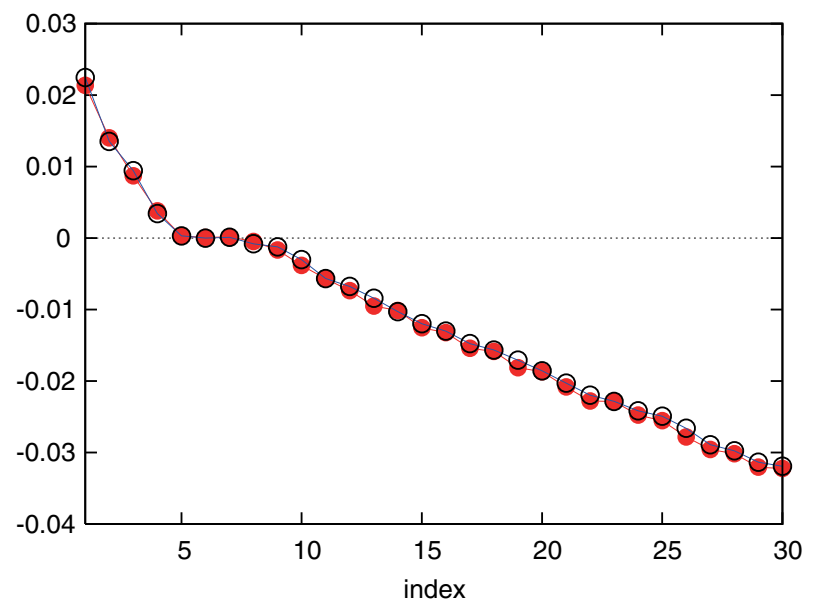

FIG. 4. (Color online) Lyapunov spectrum with different truncation numbers. Red filled circles are the $8 \times 8 \times 32$ case and black open circles are the $10 \times 10 \times 36$ case.

the Chebyshev-polynomial expansion in the wall-normal $(z)$ direction. The truncation numbers are set to be $8 \times 8 \times 32$ ( $x, y, z$ directions), and the computational grid points are $32 \times 32 \times 33$. This settings are similar to that of Kawahara and Kida's 2001 JFM paper [19] and Kawahara's 2009 paper [18]. In Fig. 4, we show a numerical convergence of the Lyapunov exponents by varying the truncation numbers. Here we compare the Lyapunov exponents calculated with the truncation number $8 \times 8 \times 32$ (red filled circles in Fig. 4) and $10 \times 10 \times 36$ (black open circles in Fig. 4) with the same averaging time $(T=23000)$. The degree of freedom as a dynamical system is 19074 (the $8 \times 8 \times 32$ case) and 32634 (the $10 \times 10 \times 36$ case), and hence the degree of freedom of the latter case is about 1.7 times larger than that of the former case. In Fig. 3, the red line is the $8 \times 8 \times 32$ case and the blue line is the $10 \times 10 \times 36$ case. Although there are some difference between these two cases [the maximum Lyapunov exponent is 0.021 (the $8 \times 8 \times 32$ case) and 0.022 (the $10 \times 10 \times 36$ case)], the quantitative features (such as the number of the positive Lyapunov exponents) are same. Therefore, we concluded that the truncation numbers $8 \times 8 \times 32$ and the computational grid points $32 \times 32 \times 33$ are sufficient for the purpose of this paper.

\section{TURBULENT BEHAVIOR OF THE MINIMAL COUETTE TURBULENCE}

Here we see the turbulent behavior of minimal Couette flow briefly. First of all, we show typical time evolution of the flow field of a single regeneration cycle. Figures 5(a)-5(f) are snapshots of the streamwise velocity field $u_{x}(\boldsymbol{x}, t)$ (contour lines) and the streamwise vorticity field $\omega_{x}(\boldsymbol{x}, t)$ (tone levels) at $t=$ (a) 2730, (b) 2750, (c) 2760, (d) 2770, (e) 2790, and (f) 2820. The upper panel of each snapshot is a cross-sectional view taken along $z=0$ and the lower one is a cross-sectional view taken along $x=1.5$ [indicated by a small arrow in Fig. 5(a)].

The regeneration cycle can be observed in these snapshots. At the beginning of the cycle $(t=2730)$, the flow field is almost $x$-independent. Particularly, we can observe the streaks 
(a)
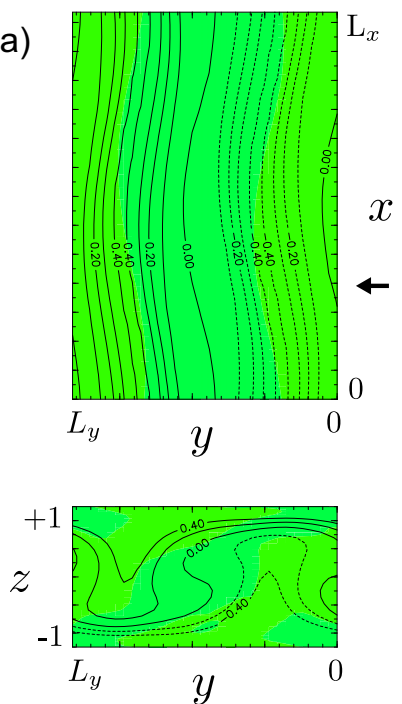

(d)
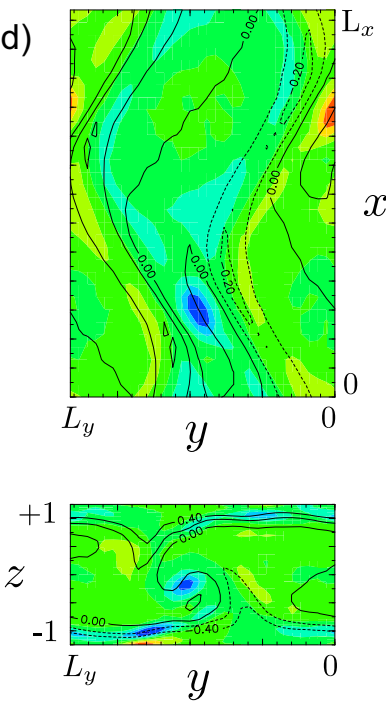

(b)
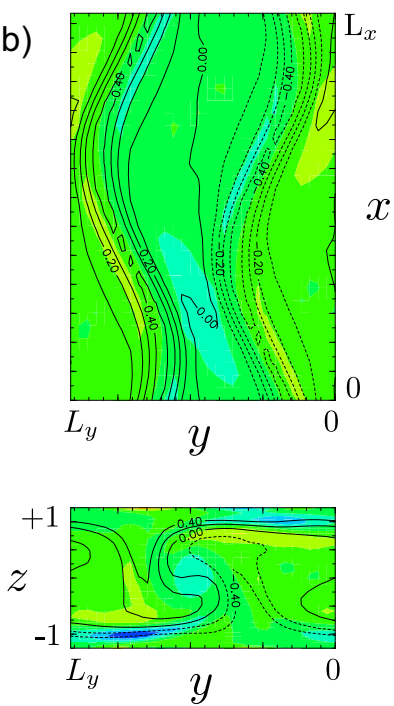

(e)
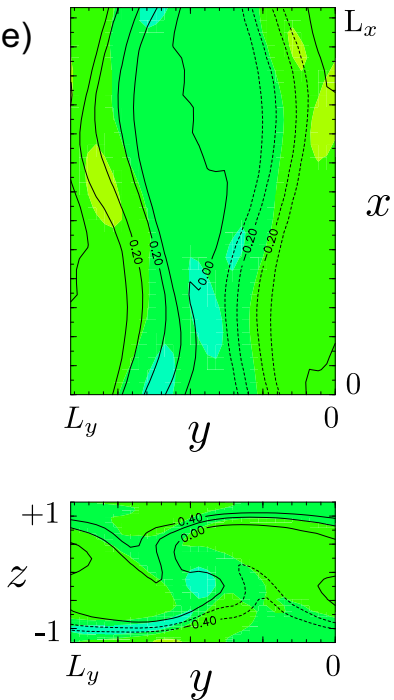

(c)

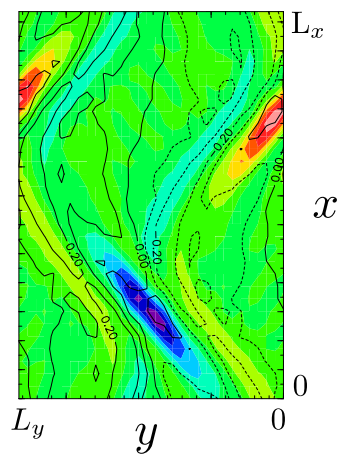

$x$

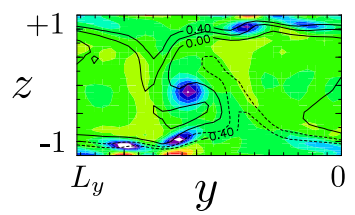

(f)

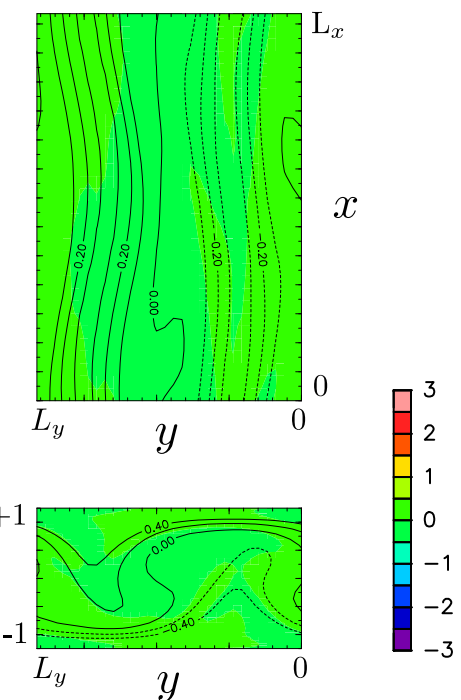

FIG. 5. (Color online) Snapshots of the streamwise velocity field $u_{x}(\boldsymbol{x}, t)$ (contour lines) and the streamwise vorticity field $\omega_{x}(\boldsymbol{x}, t)$ (tone levels) at (a) $t=2730$, (b) $t=2750$, (c) $t=2760$, (d) $t=2770$, (e) $t=2790$, (f) $t=2820$. The upper figure of each snapshot is a cross-sectional view taken along $z=0$ and the lower one is a cross-sectional view taken along $x=1.5$ [indicated by a small arrow in (a)].

which are $x$-independent flow structures consisting of an upward shift of the low streamwise velocity region and a downward shift of the high streamwise velocity region [see a contour line defined by $u_{x}=0$ in the lower panel of Fig. 5(a)]. The (high and low speed) streaks are well known as one of the key structures in understanding of the regeneration cycle. The $x$-independent streak structures soon break down and start to meander [see Figs. 5(b) and 5(c)], where by the term "break down" we mean the appearance of the small-scale structures as in Hamilton et al. [12]. In a narrow region between the meandering structures of the streaks, plus and minus strongly localized streamwise vortices appear along the near $z=0$ plane at $t=2760$ [see Fig. 5(c)]. After the disappearing of the streamwise vortices, the $x$-independent streaks are reformed [see Figs. 5(d)—5(f)], which closes the regeneration cycle.

The regeneration cycle is often studied by modal rms velocities $\sqrt{\left\langle|\hat{\boldsymbol{u}}(\boldsymbol{k}, z)|^{2}\right\rangle_{z}}$ where $\boldsymbol{k}=(k, l) \in \mathbb{Z}^{2}$ and $\hat{\boldsymbol{u}}(\boldsymbol{k}, z)=$ $\hat{\boldsymbol{u}}(k, l, z)$ is the Fourier coefficient of the velocity field: $\hat{\boldsymbol{u}}(\boldsymbol{k}, z)=$ $\left\langle\boldsymbol{u}(x, y, z) e^{-i\left(k_{x} x+k_{y} y\right)}\right\rangle_{H}\left(k_{x}=\alpha k, k_{y}=\beta l\right)$. Hereafter we drop the $z$ dependence of $\hat{\boldsymbol{u}}(\boldsymbol{k}, z)$ as $\hat{\boldsymbol{u}}(\boldsymbol{k})$ for simplicity. Figure 6 shows time series of the modal rms velocities for (a) multiple regeneration cycles $(2500 \leqslant t \leqslant 3500)$ and (b) a single regeneration cycle $(2730 \leqslant t \leqslant 2830)$, where solid lines (red) show $\boldsymbol{k}=(0,1)$, dotted lines (blue) show $\boldsymbol{k}=(1,0)$, dashed dotted lines (green) show $\boldsymbol{k}=(1,1)$, thin solid lines (pink) show $\boldsymbol{k}=(2,0)$, and dashed double-dotted lines (light blue) show $\boldsymbol{k}=(1,2)$. As reported by Hamilton et al. [12], the modal rms velocities oscillate nearly periodically and the period of the oscillation $T_{\text {cycle }}$ is $T_{\text {cycle }} \simeq 100$.

The time series in Fig. 6(b) corresponds to those of the regeneration cycle shown in Fig. 5. At the initial stage of the regeneration cycle $(t \sim 2730)$, the amplitude of $x$-independent mode is $\sqrt{\left\langle\left|\hat{\boldsymbol{u}}\left(\boldsymbol{k}_{s}\right)\right|^{2}\right\rangle_{z}}$ where $\boldsymbol{k}_{s}=(0,1)$ is the local maximum in the cycle, which corresponds to the predominance of the 
(a)

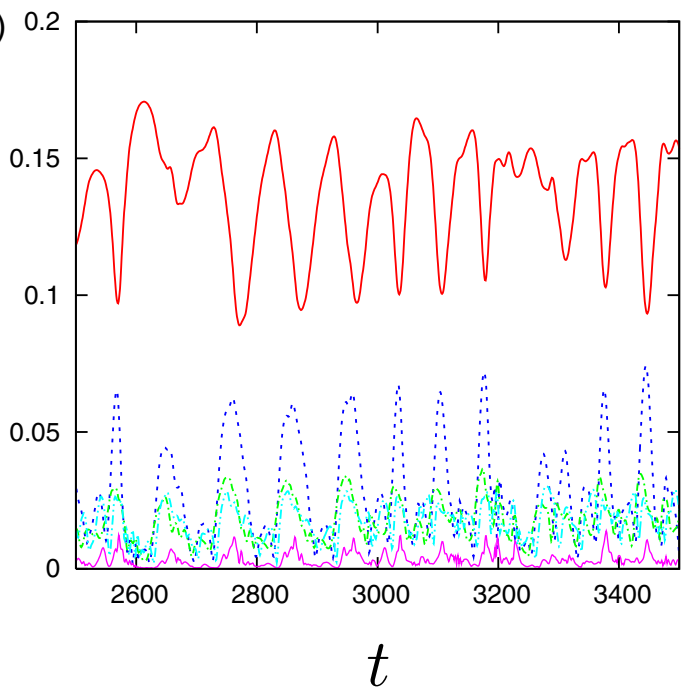

(b)

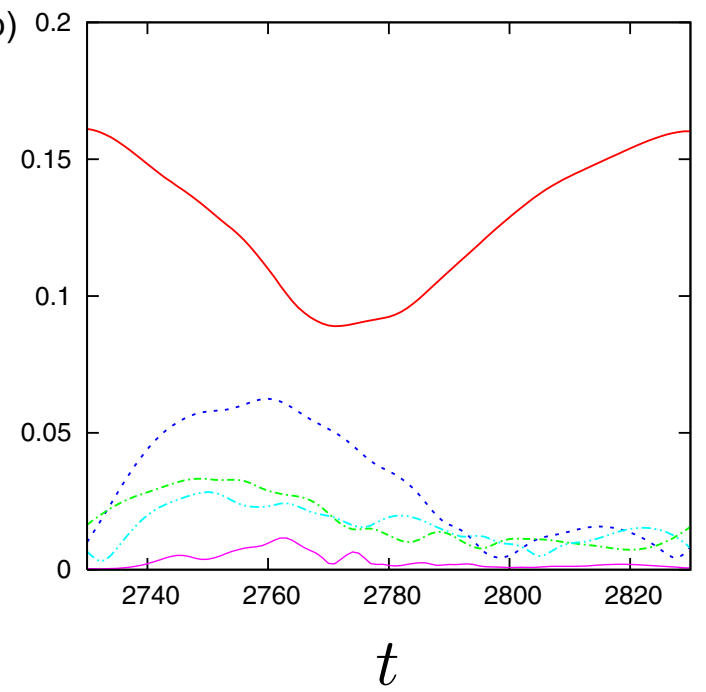

$(0,1)-$

$(1,0)$

$(1,1)$

$(2,0)$

FIG. 6. (Color online) Time series of model rms velocities $\sqrt{\left\langle|\hat{\boldsymbol{u}}(\boldsymbol{k})|^{2}\right\rangle_{z}}$ for (a) multiple regeneration cycles $(2500 \leqslant t \leqslant 3500)$ and (b) single regeneration cycle $(2730 \leqslant t \leqslant 2830)$. Solid lines (red): $\boldsymbol{k}=(0,1)$ (streak mode), dotted lines (blue): $\boldsymbol{k}=(1,0)$ (meandering mode), dashed dotted lines (green): $\boldsymbol{k}=(1,1)$, thin solid lines (pink): $\boldsymbol{k}=(2,0)$, and dashed double-dotted lines (light blue): $\boldsymbol{k}=(1,2)$.

$x$-independent streaks in the flow field as in Fig. 5(a). We refer to this $x$-independent mode as the "streak mode." The amplitude of the streak mode starts to decrease when that of the $x$-dependent mode, $\sqrt{\left\langle\left|\hat{\boldsymbol{u}}\left(\boldsymbol{k}_{m}\right)\right|^{2}\right\rangle_{z}}$ where $\boldsymbol{k}_{m}=(1,0)$, starts to increase, which corresponds to the streaks meandering as in Figs. 5(b) and 5(c). We refer to this $x$-dependent mode as the "meandering mode." After the amplitude of the meandering mode attains its local maximum $(t \sim 2760)$, that of the streak mode increases, which corresponds to the reformation of the streaks as in Figs. 5(d) and 5(f). Finally, the amplitude of the streak mode attains its local maximum again $(t \sim 2830)$, which closes the regeneration cycle.

In order to characterize the strong spatiotemporal localization of the streamwise vorticity, we show time series of "horizontal" rms of the streamwise vorticity $\sqrt{\left\langle\omega_{x}^{2}\right\rangle_{H}}$ at $z=0$ (midplane) in Fig. 7. When the $x$-independent streaks start to meander $(t \sim 2730)$, the horizontal $\mathrm{rms}$ of the streamwise vorticity $\sqrt{\left\langle\omega_{x}^{2}\right\rangle_{H}}$ starts to increase. Moreover, we can observe a sharp peak of the horizontal rms of the streamwise vorticity $\sqrt{\left\langle\omega_{x}^{2}\right\rangle_{H}}$ just before $t=2760$, indicating the strong localization of the streamwise vortices along midplane. Almost at the same time, the modal rms velocities except the streak mode reach their local maximum values (see Fig. 6).

In this paper, we divide the regeneration cycle into phase (i) and phase (ii) according to before and after the time of the sharp peak in Fig. 7: phase (i) is "streak meandering phase" (before the peak) and phase (ii) is a "streak reformation phase" (after the peak). For instance, phase (i) [resp. phase (ii)] of the regeneration cycle in Fig. 7 is $2730 \lesssim t \lesssim 2760$ (resp. $2760 \lesssim t \lesssim 2830$ ).

\section{ORBITAL INSTABILITY OF THE MINIMAL COUETTE TURBULENCE}

In this section, we study orbital instability of the regeneration cycle in the minimal Couette turbulence toward characterizing it on the basis of the full Navier-Stokes equation. In particular, by employing the covariant Lyapunov analysis [1], we show the Lyapunov spectrum, local Lyapunov exponents, and associated Lyapunov modes of the minimal Couette turbulence. As mentioned in Sec. I and Sec. II, the covariant Lyapunov modes represent intrinsic (normindependent) unstable directions along the solution orbit, which are expected to extract the instability mechanisms of turbulent flows.

\section{A. Lyapunov spectrum}

Figure 8 shows the Lyapunov spectrum $\lambda_{j}(j=$ $1,2, \ldots, 30)$ of the minimal Couette turbulence. It is found that the minimal Couette turbulence possesses four positive Lyapunov exponents $\left[\lambda_{j}>0(j=1,2,3,4)\right]$, three zero Lyapunov exponents $\left[\lambda_{j}=0(j=5,6,7)\right]$. The number of the zero Lyapunov exponents is reflected by the time translational

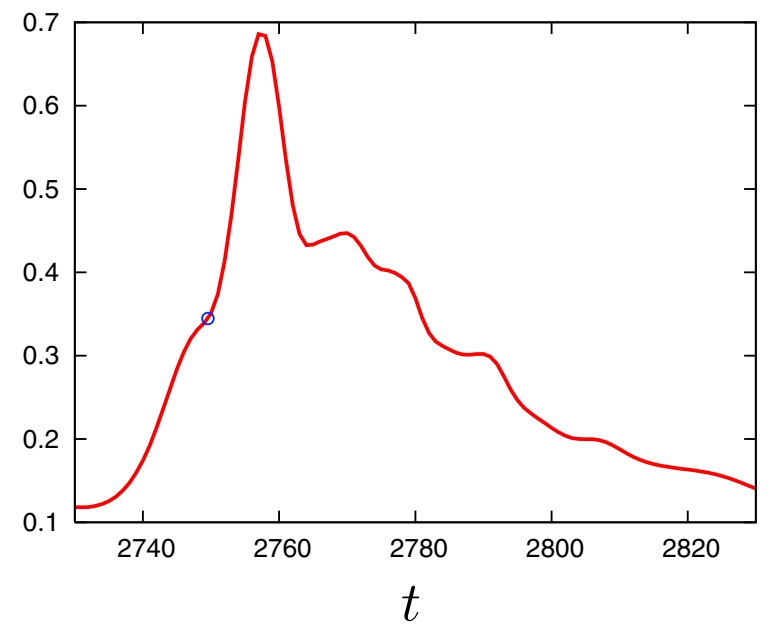

FIG. 7. (Color online) Time series of "horizontal" rms of streamwise vorticity $\sqrt{\left\langle\omega_{x}^{2}\right\rangle_{H}}$ of the single regeneration cycle at the midplane $(z=0)$. Blue circle in the figure represents the value of $\sqrt{\left\langle\omega_{x}^{2}\right\rangle_{H}}$ at $t=2750$ for reference in a later section. 


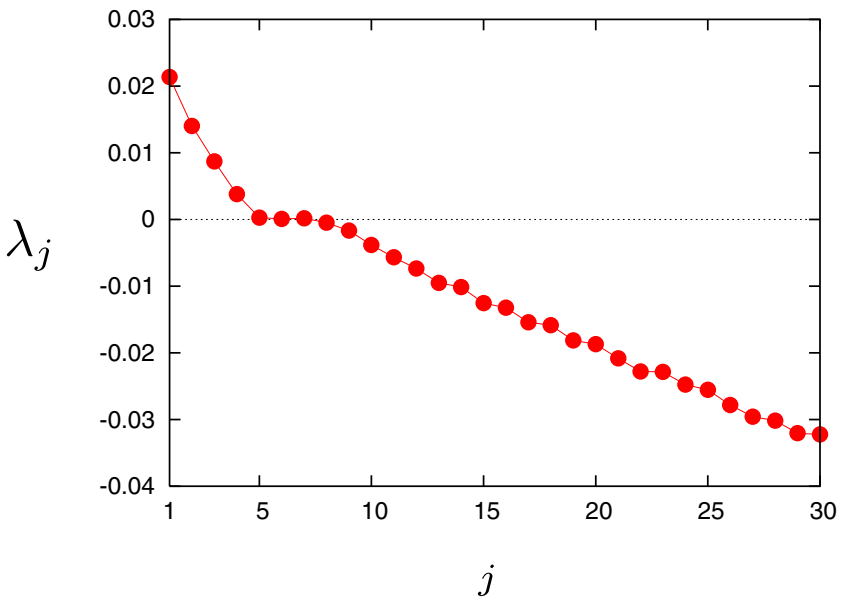

FIG. 8. (Color online) Lyapunov spectrum $\quad \lambda_{j}(j=$ $1,2,3, \ldots, 30)$ of the minimal Couette turbulence. The minimal Couette turbulence possesses four positive Lyapunov exponents $\left[\lambda_{j}>0(j=1,2,3,4)\right]$ and three zero Lyapunov exponents $\left[\lambda_{j}=0(j=5,6,7)\right]$. The maximum Lyapunov exponent is $\lambda_{1}=0.021$.

symmetry and the spatial translational symmetries in horizontal directions ( $x$ and $y$ directions). The maximum Lyapunov exponent $\lambda_{1}$ is $\lambda_{1}=0.021$. Interestingly, the maximum Lyapunov exponent is close to the maximum Floquet exponent $\mu=0.019$ of the "strong" UPO, whose statistics approximate well the statistics of turbulence, as reported by Kawahara [18]. Besides, the Floquet exponents of unstable (relative) periodic orbits calculated by Viswanath [21] are also near the maximum Lyapunov exponent $(\mu=0.023 \sim 0.035$; see Table I in Viswanath [21]). The Lyapunov (Kaplan-Yorke) dimension $D_{L}\left(=K+\frac{1}{\left|\lambda_{K+1}\right|} \sum_{j=1}^{K} \lambda_{j}\right)$ is $D_{L}=14.8$ where $K$ is the largest integer such that $\sum_{j=1}^{K} \lambda_{j} \geqslant 0$ (Fig. 9). The Kolmogorov-Sinai entropy estimated by summation of the positive Lyapunov exponents is $h_{\mathrm{KS}}=\sum_{j=1}^{4}=0.048$.

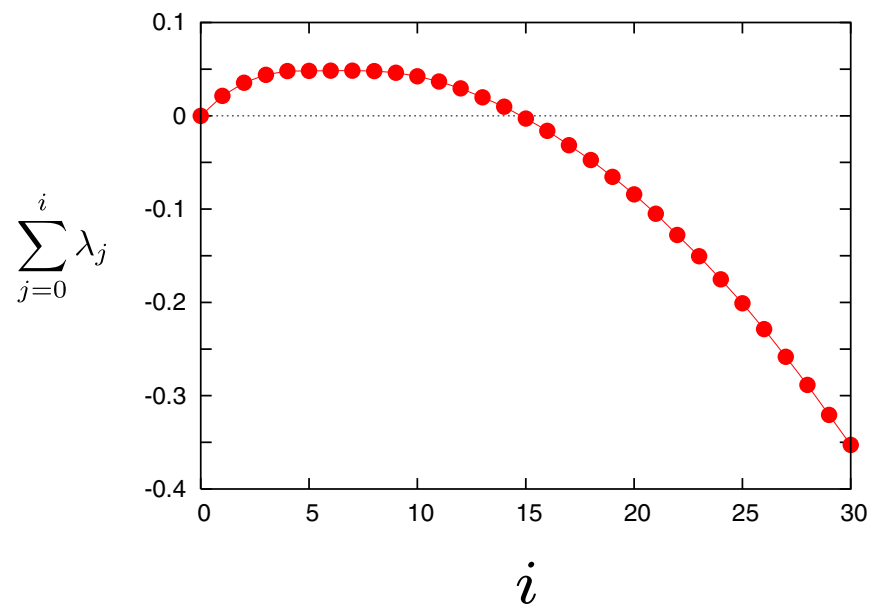

FIG. 9. (Color online) Summation of the Lyapunov exponents $\sum_{j=0}^{i} \lambda_{j}$ of the minimal Couette turbulence. The Lyapunov dimension is $D_{L}=14.8$ and Kolmogorov-Sinai entropy is $h_{\mathrm{KS}}=0.048$.

\section{B. Local Lyapunov exponents}

We here study the instability mechanisms in the regeneration cycle via local Lyapunov exponents $\tilde{\lambda}_{j}(t, \tau)$ (sometimes referred to as "finite time" Lyapunov exponents). The local Lyapunov exponent at a state point $\boldsymbol{x}(t)$ is the logarithmic growth rate of the Lyapunov vector $\boldsymbol{y}^{(j)}(t)$ during $\tau$, which is defined by

$$
\tilde{\lambda}_{j}(t, \tau)=\frac{1}{\tau} \ln \frac{\left\|\boldsymbol{y}^{(j)}(t+\tau)\right\|}{\left\|\boldsymbol{y}^{(j)}(t)\right\|},
$$

where $\tau$ denotes a local average time and here we set $\tau=1$. We obtained the local Lyapunov exponents by calculating the ratio of the norm of the Lyapunov vectors $\left\|\boldsymbol{y}^{(j)}(t+\tau)\right\| /\left\|\boldsymbol{y}^{(j)}(t)\right\|$ along the chaotic orbit. As $\tau \rightarrow \infty$, the local Lyapunov exponent converges to the Lyapunov exponent defined by Eq. (6), i.e., $\lim _{\tau \rightarrow \infty} \tilde{\lambda}_{j}(t, \tau)=\lambda_{j}$, which is independent of $\boldsymbol{x}(t)$. The upper panel of Fig. 10 shows the time series of the local Lyapunov exponents $\tilde{\lambda}_{j}(j=1,2,3,4)$ associated with the positive Lyapunov exponents for $j=1$ (red solid line), 2 (green dotted line), 3 (blue dashed-dotted line), and 4 (pink dashed double-dotted line) during $2730 \leqslant t \leqslant 3030$ including three regeneration cycles. Roughly speaking, the periods of the fluctuations of the local Lyapunov exponents are $10 \sim 20$ which indicates that the unstable mode of the flow is sensitive to the details of the base flow as observed in Hamilton et al. [12]. Corresponding to the rms streamwise vorticity $\sqrt{\left\langle\omega_{x}^{2}\right\rangle_{H}}$ (the lower panel of the Fig. 10), it is found that the local Lyapunov exponent tends to be positive during phase (i) (e.g., $2730 \leqslant t \leqslant 2760$ ) and tends to be zero or negative during phase (ii) (e.g., $2760 \leqslant t \leqslant 2830$ ). Furthermore, with the shifts from phase (i) to phase (ii), the local Lyapunov exponents rapidly decrease from positive values to negative values, and the spatiotemporal localization of the streamwise vorticity gives the largest local Lyapunov exponent. These observations suggest that the streamwise vortices have a key influence on the instability of the minimal Couette turbulence: the flow is unstable during phase (i) and neutral or stable during phase (ii).

To show the difference between phase (i) and phase (ii) more clearly, we calculate a finite time growth rate $\Lambda_{j}\left(t_{0}, \tau\right)$ of the Lyapunov vector defined by

$$
\Lambda_{j}\left(t_{0}, \tau\right)=\frac{\left\|\boldsymbol{y}^{(j)}\left(t_{0}+\tau\right)\right\|}{\left\|\boldsymbol{y}^{(j)}\left(t_{0}\right)\right\|} .
$$

Here, we fix the initial time $t_{0}$, and consider $\Lambda_{j}\left(t_{0}, \tau\right)$ as a function of $\tau$. Figure 11 shows the finite time growth rate $\Lambda_{j}\left(t_{0}, \tau\right)$ where (a) $t_{0}=2730$ [onset of phase (i)], and (b) $t_{0}=2760$ [onset of phase (ii)] for $j=1,2,3$,4. In Fig. 11(a), clearly the small perturbation grows until $\tau \simeq 30$ (i.e., $t_{0}+$ $\tau=2760$ ), and the perturbation growth reaches the maximum when the streamwise vortices localize strongly in space and time. After the perturbation growth reaches the maximum, the small perturbation does not grow since the finite-time growth rate is $\Lambda_{j}\left(t_{0}, \tau\right) \lesssim 1$ as shown in Fig. 11(b), suggesting that there is no exponential instability in phase (ii).

\section{Lyapunov vectors}

The local Lyapunov exponents indicate that the minimal Couette turbulence is unstable only in phase (i). Here we see 


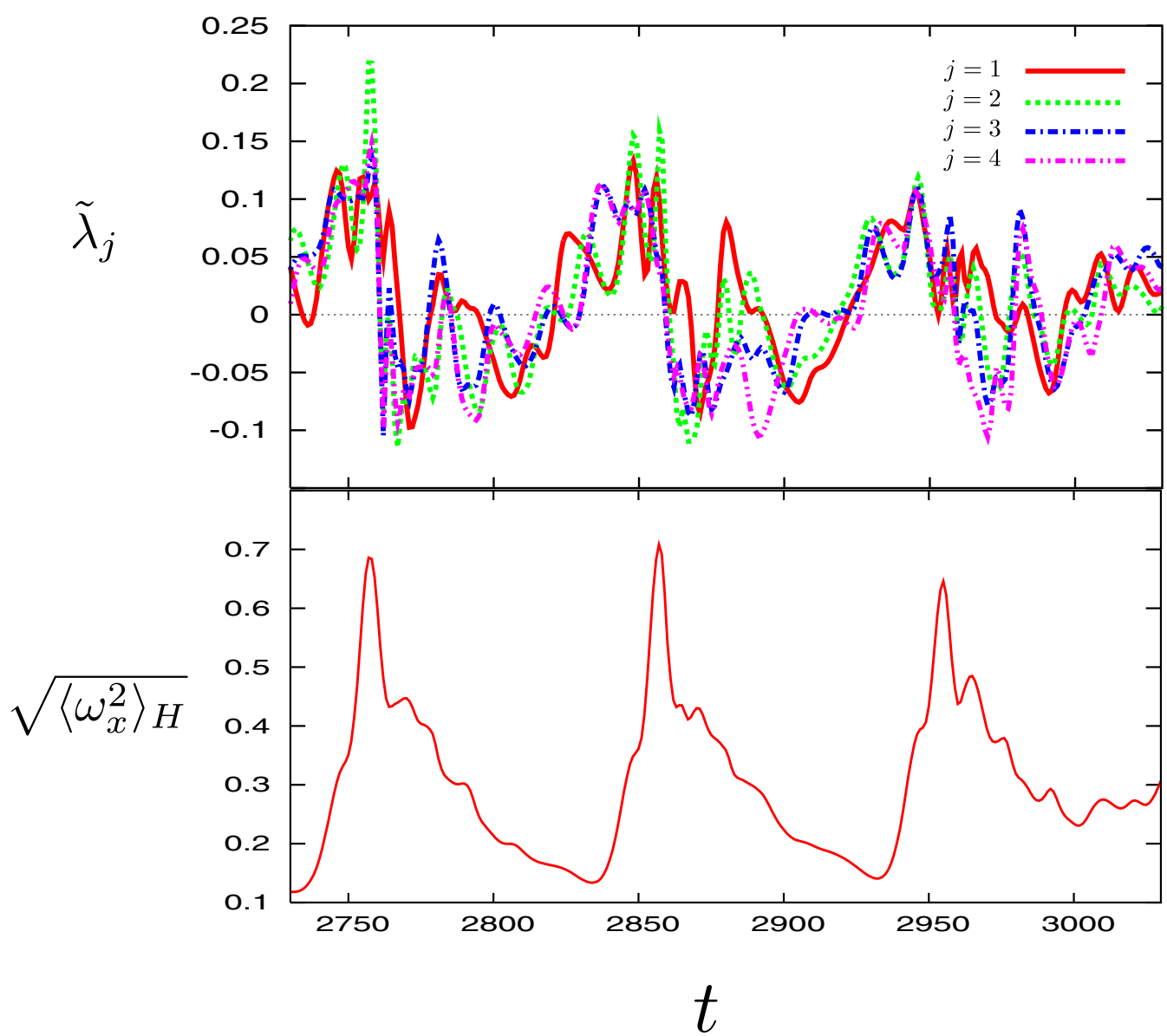

FIG. 10. (Color online) Upper panel: Time series of local Lyapunov exponents $\tilde{\lambda}_{j}(t)$ for (solid line; red) $j=1$, (dotted line; green) $j=2$, (dashed dotted; blue) $j=3$, and (dashed double-dotted; pink) $j=4$. Lower panel: The "horizontal" rms of streamwise vorticity $\sqrt{\left\langle\omega_{x}^{2}\right\rangle} H$ at the midplane $(z=0)$.

the Lyapunov vectors (modes) associated with the positive Lyapunov exponents, which are expected to carry important information on the flow instability in phase (i).

First, we show the Lyapunov modes at the initial stage of phase (i) $(t=2730)$ in Fig. 12. The streamwise vorticity of the Lyapunov modes $\delta \omega_{x j}$ is shown as color tone for $j=1,2,3,4$

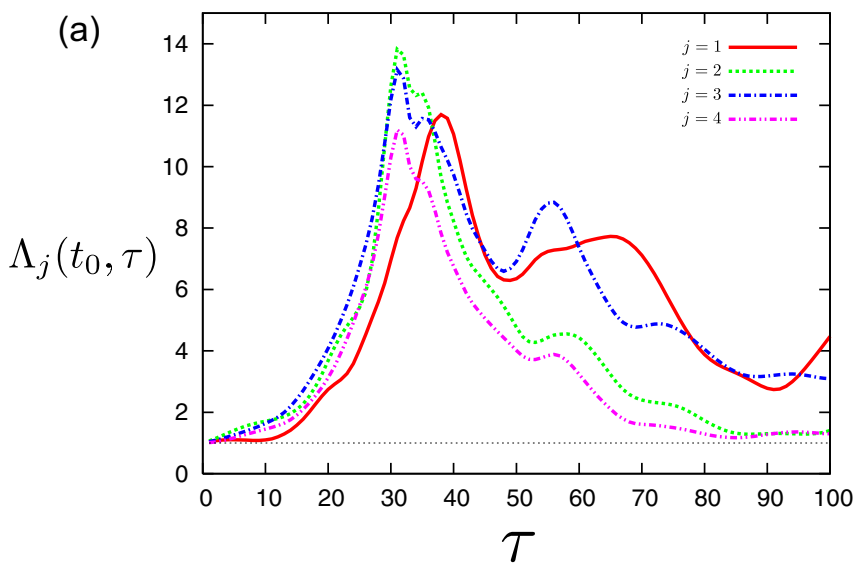

in Figs. 12(a)-12(d), respectively, and streamwise velocity of the base flow (the minimal Couette turbulence) is shown as counter lines. The Lyapunov modes are normalized by the enstrophy norm as $1 / 2\left\langle\left|\delta \omega_{j}\right|^{2}\right\rangle_{V}=1$. The upper figure of each panel is a cross-sectional view taken along $z=0.8$ and the lower one is a cross-sectional view taken along $x=1.5$. The

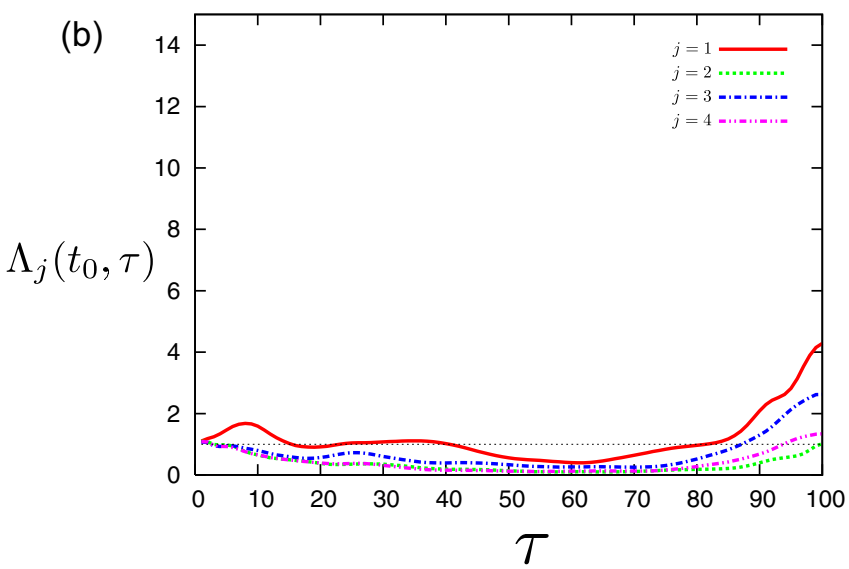

FIG. 11. (Color online) Finite-time growth rate $\Lambda\left(t_{0}, \tau\right)$, (a) $t_{0}=2730$, (b) $t_{0}=2760$, for $\tilde{\lambda}_{j}(t)$ for (solid line; red) $j=1$, (dotted line; green) $j=2$, (dashed dotted; blue) $j=3$, and (dashed double-dotted; pink) $j=4$. The black dot horizontal line denotes $\Lambda_{j}\left(t_{0}, \tau\right) \equiv 1$ (i.e., neutral). 
(a)
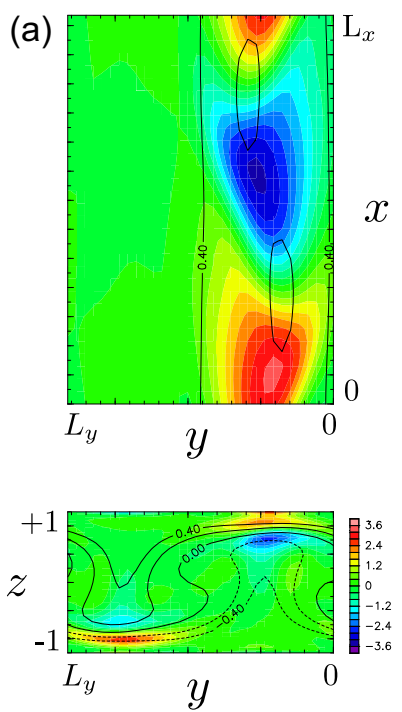

(b)
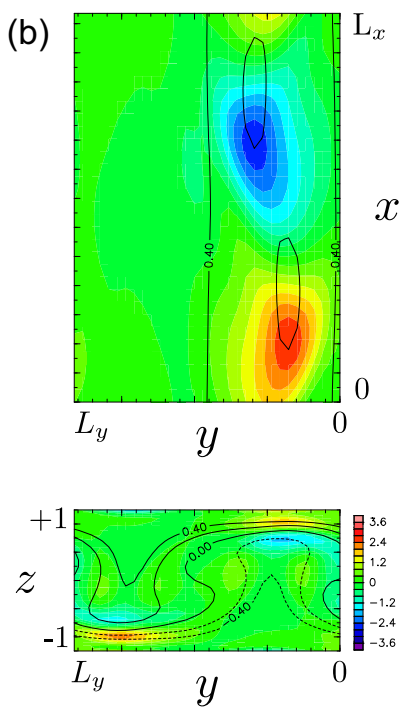

(c)
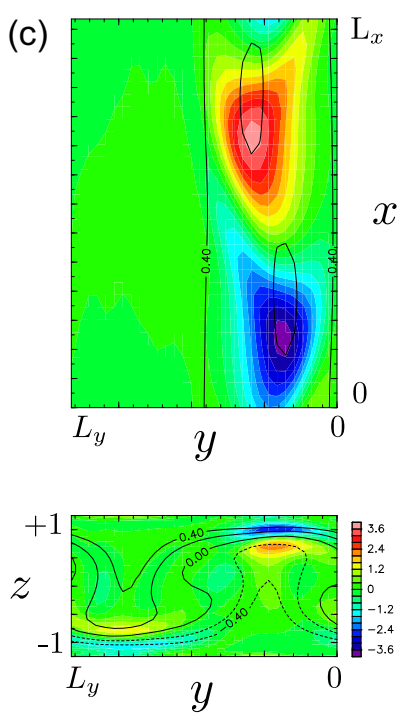

(d)
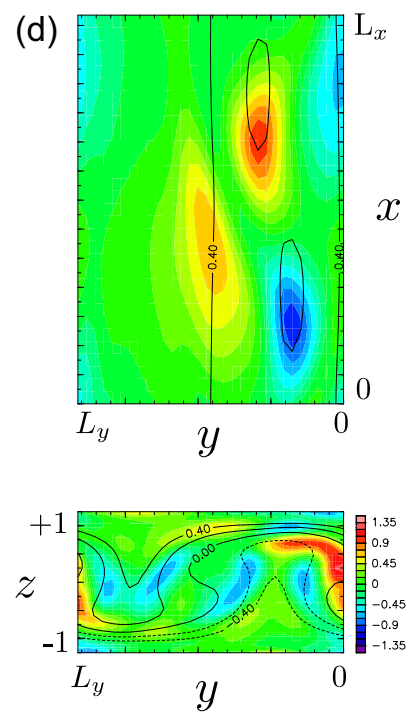

FIG. 12. (Color online) Lyapunov modes at the initial stage of the phase (i) $(t=2730)$. Streamwise vorticities of the Lyapunov modes $\delta \omega_{x j}$ are shown as color tone for (a) $j=1$, (b) $j=2$, (c) $j=3$, (d) $j=4$, and streamwise velocities of the base flow are shown as counter lines.

streamwise vorticity of the Lyapunov mode $\delta \omega_{x j}(j=1,2,3)$ is found to be localized at near-wall regions of the $u_{x}=0$ sheet in the cross-stream plane (lower panel) and the signs of the vorticity appear alternately along the streamwise direction (upper panel). Figure 13 shows the cross-sectional views of the first (i.e., the most unstable) Lyapunov mode at the same time ( $t=2730$ ) along the streamwise direction: (a) $x=L_{x} / 4$, (b) $x=L_{x} / 2$, (c) $x=3 L_{x} / 4$, (d) $x=L_{x}$. Streamwise vorticity of the Lyapunov mode $\delta \omega_{x 1}$ is shown as color tone and streamwise velocity of the base flow is shown as counter lines. These patterns of the Lyapunov modes at the initial stage of phase (i) are similar to that of the eigenfunctions (sinuous streak instability modes) as a result of linear instability analysis of the model streak calculated by Schoppa and Hussain [16] and the corrugated sheet calculated by Kawahara et al. [17]. Particularly, we can see the characteristics of the sinuous instability mode in the patterns of the Lyapunov modes, i.e., (A) appearances of different signs streamwise vorticity alternatively along the streamwise direction, and (B) localizations of streamwise vorticity near the low-speed streak "crest" and the high-speed "trough" regions. Hence we conclude that the Lyapunov modes at the initial stage of phase (i) correspond to the sinuous modes of the model streaks which make the $x$-independent streak meander. It is worth noting that the Lyapunov modes $\delta \omega_{x j}(j=1,2,3,4)$ are unstable disturbances in an asymptotic sense, while the sinuous modes of the model streaks are an unstable disturbance considering the model streaks as stationary solutions. Thus, it is found that the sinuous instability mode is important in the asymptotic instability of the turbulent solution.

The growth of the sinuous disturbance makes the streaks meander, and then the streamwise vortices localize strongly at the midplane, which gives the largest local Lyapunov exponents. In Fig. 14, we show the Lyapunov modes at the (a)

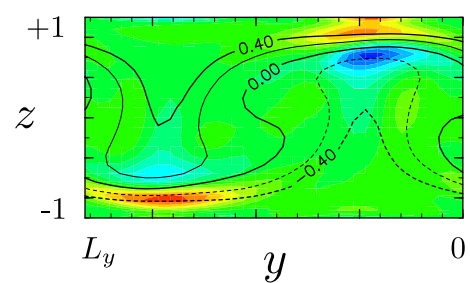

(c)

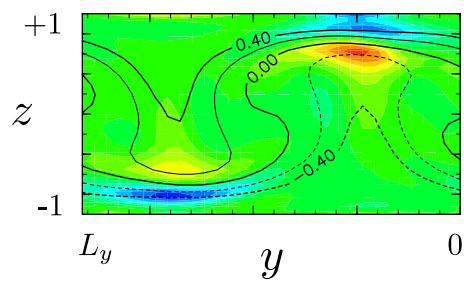

(b)

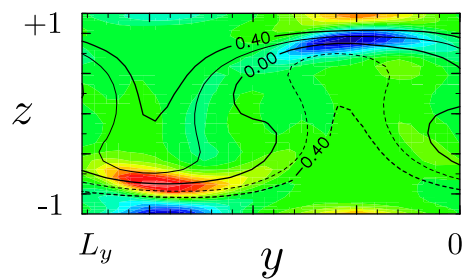

(d)

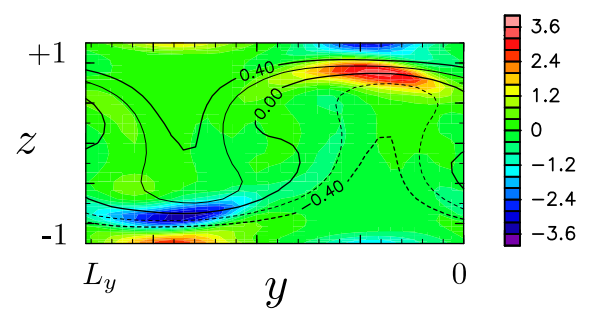

FIG. 13. (Color online) Cross-sectional views of the most unstable Lyapunov mode at the initial stage of the phase (i) $(t=2730)$ at (a) $x=L_{x} / 4$, (b) $x=L_{x} / 2$, (c) $x=3 L_{x} / 4$, (d) $x=L_{x}$. Streamwise vorticities of the Lyapunov mode $\delta \omega_{x 1}$ are shown as color tone and streamwise velocities of the base flow are shown as counter lines. 
(a)
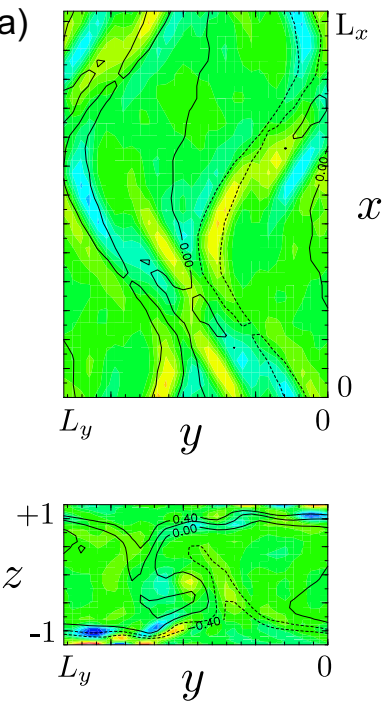

(b)
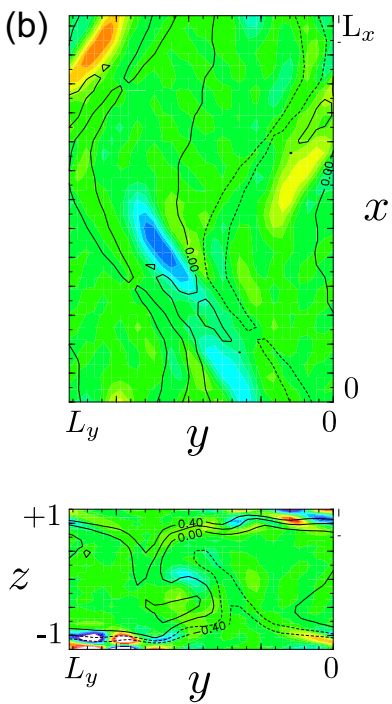

(c)
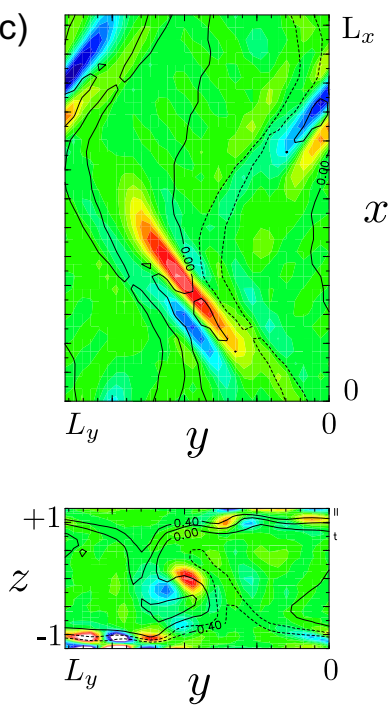

(d)
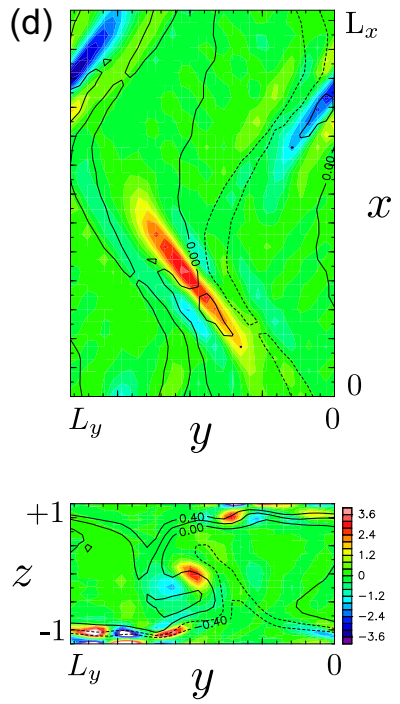

FIG. 14. (Color online) Lyapunov modes at the final stage of the phase (i) $(t=2760)$. Streamwise vorticity of the Lyapunov mode $\delta \omega_{x j}$ is shown as color tone for (a) $j=1$, (b) $j=2$, (c) $j=3$, (d) $j=4$, and streamwise velocity of the base flow is shown as counter lines.

final stage of the phase (i) which are associated with the largest local Lyapunov exponents. The streamwise vorticity of the Lyapunov modes $\delta \omega_{x j}$ is shown as color tone for (a) $j=1$, (b) $j=2$, (c) $j=3$, (d) $j=4$, and streamwise velocity of the base flow is shown as counter lines as in Fig. 12. The Lyapunov modes are normalized by the enstrophy norm as $1 / 2\left\langle\left|\delta \boldsymbol{\omega}_{j}\right|^{2}\right\rangle_{V}=1$. The upper figure of each panel is a cross-sectional view taken along $z=0$ (midplane) and the lower one is the cross-sectional view taken along $x=1.5$. It is found that the streamwise vorticity of the Lyapunov mode $\delta \omega_{x j}$ (particularly $j=3,4$ ) localizes at the narrow region between the meandering streaks where the streamwise vorticity of the base flow also localizes as seen in Fig. 5(c). This observation supports the idea that the flow instability at the final stage of the phase (i) originates from the spatiotemporal localization of the streamwise vortices at the midplane.

In this section, we found that the local Lyapunov exponents and the associated Lyapunov modes indicate the two types of instabilities in phase (i): the sinuous instability and the instability related to the localization of streamwise vortices (hereafter we refer to it as streamwise vortices instability). Thus, instabilities dominate over phase (i). Moreover, the covariant Lyapunov analysis also indicates that there is no instability in phase (ii). Here a natural question arises: What drives phase (ii)? In particular, what reforms the streaks? In the next section, we discuss the streak formation by using energy budget analysis.

\section{ENERGY BUDGET ANALYSIS OF THE MINIMAL COUETTE TURBULENCE}

\section{A. What reforms the streaks?}

Here we discuss the reformation of the ( $x$-independent) streaks. We consider the reformation process of the streaks as an energy gain process of the streak mode $\hat{\boldsymbol{u}}\left(\boldsymbol{k}_{s}\right)$ where $\boldsymbol{k}_{s}=(0,1)$ whose amplitude increases in the phase (ii) shown in the time series of the modal rms velocities (Fig. 6).
An evolution equation of "modal energy" of the $\boldsymbol{k}$ mode is generally

$$
\begin{aligned}
\frac{d}{d t}\left\langle|\hat{\boldsymbol{u}}(\boldsymbol{k})|^{2}\right\rangle_{z}= & -\left\langle 2 \operatorname{Re}\left[\hat{\boldsymbol{u}}^{*}(\boldsymbol{k}) \cdot\left(\hat{u}_{z}(\boldsymbol{k}) \partial_{z}\right) \hat{\boldsymbol{u}}(\mathbf{0})\right]\right\rangle_{z} \\
& -\left\langle\sum_{\substack{\boldsymbol{k}^{\prime \prime}+\boldsymbol{k}^{\prime}=\boldsymbol{k}, \boldsymbol{k}^{\prime} \neq \mathbf{0}, \boldsymbol{k}^{\prime \prime} \neq \mathbf{0}}} \mathcal{N}\left(\hat{\boldsymbol{u}}(\boldsymbol{k}), \hat{\boldsymbol{u}}\left(\boldsymbol{k}^{\prime}\right), \hat{\boldsymbol{u}}\left(\boldsymbol{k}^{\prime \prime}\right)\right)\right\rangle_{z} \\
& -\frac{2}{\operatorname{Re}}\left\langle\left\{(\alpha k)^{2}+(\beta l)^{2}\right\}|\hat{\boldsymbol{u}}(\boldsymbol{k})|^{2}+\left|\partial_{z} \hat{\boldsymbol{u}}(\boldsymbol{k})\right|^{2}\right\rangle_{z} .
\end{aligned}
$$

The first term of the right-hand side of the above equation is the mean flow interaction term, the second term is other nonlinear terms (triad interaction $\boldsymbol{k}_{s}=\boldsymbol{k}^{\prime}+\boldsymbol{k}^{\prime \prime}$ with $\boldsymbol{k}^{\prime}, \boldsymbol{k}^{\prime \prime}$ modes except the mean flow mode: $\boldsymbol{k}^{\prime} \neq \mathbf{0}, \boldsymbol{k}^{\prime \prime} \neq \mathbf{0}$ ), and the third term is the viscous term. Hereafter we write the mean flow interaction term as $\langle g(\boldsymbol{k}, z)\rangle_{z}=-\left\langle 2 \operatorname{Re}\left[\hat{\boldsymbol{u}}^{*}(\boldsymbol{k}) \cdot\left(\hat{u}_{z}(\boldsymbol{k}) \partial_{z}\right) \hat{\boldsymbol{u}}(\mathbf{0})\right]\right\rangle_{z}$.

In order to study the energy gain process of the streak mode, we show an energy budget analysis of the evolution equation of streak modal "energy" $\left[\boldsymbol{k}=\boldsymbol{k}_{s}\right.$ in the evolution equation (10)] in Fig. 15. The red (solid) line is the time derivative term of $\left\langle\left|\hat{\boldsymbol{u}}\left(\boldsymbol{k}_{s}\right)\right|^{2}\right\rangle_{z}$ (left-hand side of the evolution equation), the green (dashed) line is the mean flow interaction term, the blue (dot) line is other nonlinear terms, and the pink (dashed-dot) line is the viscous term, where other nonlinear terms are calculated from the other three terms. The time derivative of $\left\langle\left|\hat{\boldsymbol{u}}\left(\boldsymbol{k}_{s}\right)\right|^{2}\right\rangle_{z}$ is negative in the phase (i) and positive in the phase (ii), which corresponds to the breakdown and reformation of the streaks. Energy injection into the streak mode (i.e., the positive term on the right-hand side of the evolution equation) is only given by the mean flow interaction term (the green dashed line) throughout the regeneration cycle. Therefore, we conclude that the reformation of the streaks is based on the energy injection through the mean flow interaction. Interaction with the mean flow is the basis of streak generation in the "transient growth" mechanism associated with nonnormality of the base flow. It remains unclear whether the mechanism is the same here, as the base flow is fluctuating. 


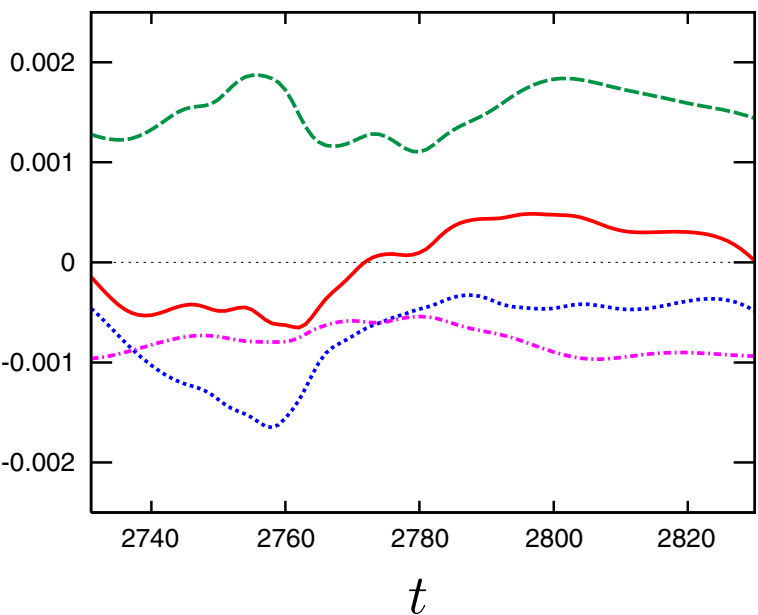

FIG. 15. (Color online) Budget analysis of the evolution equation of the "modal energy" (10) in the case of the streak mode (i.e., $\boldsymbol{k}=\boldsymbol{k}_{s}$ ). The red (solid) line is the time derivative term (left-hand side of the evolution equation), the green (dashed) line is the mean flow interaction term, the blue (dot) line is other nonlinear terms, and the pink (dashed-dot) line is the viscous term.

Here we consider the wall-normal profiles of the mean flow interaction term $g(\boldsymbol{k}, z)$. Figure 16(a) shows the wall-normal profile of the mean flow interaction term of the streak mode $g\left(\boldsymbol{k}_{s}, z\right)$ (red line with closed circles) and that of the meandering mode $g\left(\boldsymbol{k}_{m}, z\right)$ (blue line with open circles) at $t=2800$. These profiles are almost the same throughout the regeneration cycle. Furthermore, $g\left(\boldsymbol{k}_{s}, z\right)>0$ means the energy injection from the mean flow to the streak mode throughout the cycle. Figure 16(b) shows the cross-streamwise sectional view of the streamwise velocity field consisting of the streak mode only: $u_{x}^{k_{s}}(y, z)$ at $t=2820$ (color tone). The solid lines in Fig. 16(b) are the contour lines of the streamwise velocity field consisting of the all modes. The wall-normal profile of $u_{x}^{k_{s}}(y, z)$ has two local maxima at $z \simeq \pm 0.6$, which coincide with the profile of the mean flow interaction term of the streak mode $g\left(\boldsymbol{k}_{s}, z\right)$. This observation also supports our conclusion that the reformation of the streak is driven by the mean flow interaction. Moreover, the interaction between the mean flow and the meandering mode is almost zero: $g\left(\boldsymbol{k}_{m}, z\right) \simeq 0$ throughout the regeneration cycle. Therefore the energy is injected from the mean flow not to the meandering mode but to the streak mode.
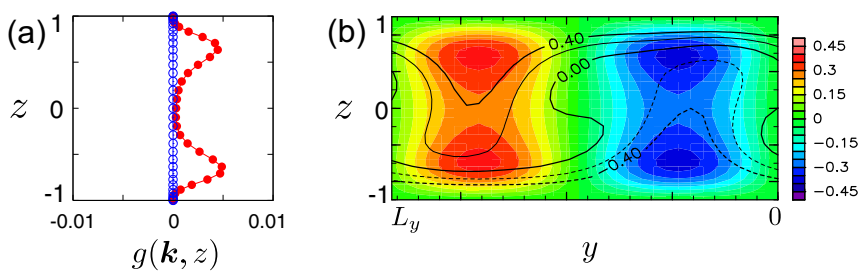

FIG. 16. (Color online) (a) Wall-normal profile of the mean flow interaction term of the streak mode $g\left(\boldsymbol{k}_{s}, z\right)$ (red line with closed circles) and the meandering mode $g\left(\boldsymbol{k}_{m}, z\right)$ (blue line with open circles) at $t=2800$. (b) The cross-sectional view of the streamwise velocity field consisting of the streak mode only, $u_{x}^{\boldsymbol{k}_{s}}(y, z)$ (color tone), and that consisting of all modes (solid lines) at $t=2820$.

\section{B. What controls the regeneration cycle?}

In the previous subsection, we concluded that the mean flow interaction reforms the streaks. Besides, the energy budget analysis (Fig. 15) shows that the mean flow interaction term and the viscous term do not change drastically throughout the cycle. In contrast, the other nonlinear terms (the blue dot line in Fig. 15) change with the shift from phase (i) to phase (ii): the other nonlinear terms reach a minimum $(\simeq-0.0015)$ in the phase (i) and maintain an almost constant value $(\simeq-0.0005)$ in the phase (ii). Thus, it appears that the other nonlinear interaction terms control the sign of the time derivative of $\left\langle\left|\hat{\boldsymbol{u}}\left(\boldsymbol{k}_{s}\right)\right|^{2}\right\rangle_{z}$, i.e., the regeneration cycle.

At the initial stage of the phase (i), the amplitude of the streak mode decreases and the amplitude of the meandering mode increases. This observation implies that the accumulated energy in the streak mode "leaks" to the meandering mode through the nonlinear interaction during the phase (i). Therefore we focus our attention on the detailed streak modal energy equation, extracting the nonlinear interaction between the streak mode $\boldsymbol{k}_{s}$ and the meandering mode $\boldsymbol{k}_{m}$ in particular:

$$
\begin{aligned}
& \frac{d}{d t}\left\langle\left|\hat{\boldsymbol{u}}\left(\boldsymbol{k}_{s}\right)\right|^{2}\right\rangle_{z}=\left\langle g\left(\boldsymbol{k}_{s}, z\right)\right\rangle_{z}+\left\langle h\left(\boldsymbol{k}_{s}, z\right)\right\rangle_{z} \\
& -\left\langle\sum_{\substack{\boldsymbol{k}^{\prime \prime}+\boldsymbol{k}^{\prime}=\boldsymbol{k}_{s}, \boldsymbol{k}^{\prime} \neq \mathbf{0}, \boldsymbol{k}^{\prime \prime} \neq \mathbf{0} \\
\boldsymbol{k}^{\prime} \neq \pm \boldsymbol{k}_{m}, \boldsymbol{k}^{\prime \prime} \neq \pm \boldsymbol{k}_{m},}} \mathcal{N}^{\prime}\left(\hat{\boldsymbol{u}}\left(\boldsymbol{k}_{s}\right), \hat{\boldsymbol{u}}\left(\boldsymbol{k}^{\prime}\right), \hat{\boldsymbol{u}}\left(\boldsymbol{k}^{\prime \prime}\right)\right)\right\rangle_{z} \\
& -\frac{2}{\operatorname{Re}}\left\langle\beta^{2}\left|\hat{\boldsymbol{u}}\left(\boldsymbol{k}_{s}\right)\right|^{2}+\left|\partial_{z} \hat{\boldsymbol{u}}\left(\boldsymbol{k}_{s}\right)\right|^{2}\right\rangle_{z} .
\end{aligned}
$$

The second term on the right-hand side $\left\langle h\left(\boldsymbol{k}_{s}, z\right)\right\rangle_{z}$ is the interaction between the streak mode and the meandering mode:

$$
\begin{aligned}
\left\langle h\left(\boldsymbol{k}_{s}, z\right)\right\rangle_{z}= & -\left\langle 2 \operatorname{Re}\left[\hat{\boldsymbol{u}}^{*}\left(\boldsymbol{k}_{s}\right) \cdot\left(i \alpha \hat{u}_{x}\left(\boldsymbol{k}_{o b-}\right)+\hat{u}_{z}\left(\boldsymbol{k}_{o b-}\right) \partial_{z}\right) \hat{\boldsymbol{u}}\left(\boldsymbol{k}_{m}\right)\right]\right\rangle_{z}-\left\langle 2 \operatorname{Re}\left[\hat{\boldsymbol{u}}^{*}\left(\boldsymbol{k}_{s}\right) \cdot\left(-i \alpha \hat{u}_{x}\left(\boldsymbol{k}_{m}\right)+i \beta \hat{u}_{y}\left(\boldsymbol{k}_{m}\right)+\hat{u}_{z}\left(\boldsymbol{k}_{m}\right) \partial_{z}\right) \hat{\boldsymbol{u}}\left(\boldsymbol{k}_{o b-}\right)\right]\right\rangle_{z} \\
& -\left\langle 2 \operatorname{Re}\left[\hat{\boldsymbol{u}}^{*}\left(\boldsymbol{k}_{s}\right) \cdot\left(-i \alpha \hat{u}_{x}\left(\boldsymbol{k}_{o b+}\right)+\hat{u}_{z}\left(\boldsymbol{k}_{o b+}\right) \partial_{z}\right) \hat{\boldsymbol{u}}^{*}\left(\boldsymbol{k}_{m}\right)\right]\right\rangle_{z}-\left\langle 2 \operatorname{Re}\left[\hat{\boldsymbol{u}}^{*}\left(\boldsymbol{k}_{s}\right) \cdot\left(i \alpha \hat{u}_{x}^{*}\left(\boldsymbol{k}_{m}\right)+i \beta \hat{u}_{y}^{*}\left(\boldsymbol{k}_{m}\right)+\hat{u}_{z}^{*}\left(\boldsymbol{k}_{m}\right) \partial_{z}\right) \hat{\boldsymbol{u}}\left(\boldsymbol{k}_{o b+}\right)\right]\right\rangle_{z},
\end{aligned}
$$

where $\boldsymbol{k}_{o b+}=(1,1)$ and $\boldsymbol{k}_{o b-}=(-1,1)$ which close the triad interaction: $\boldsymbol{k}_{s}=\boldsymbol{k}^{\prime}+\boldsymbol{k}^{\prime \prime}$. The second term $\left\langle h\left(\boldsymbol{k}_{s}, z\right)\right\rangle_{z}$ and third term on the right-hand side of Eq. (11) are the difference between the previous streak modal en- ergy equation (10) and the detailed streak modal energy equation (11).

In Fig. 17, the blue (dotted) line is the other nonlinear terms and the navy (dashed double-dotted) line is the nonlinear 


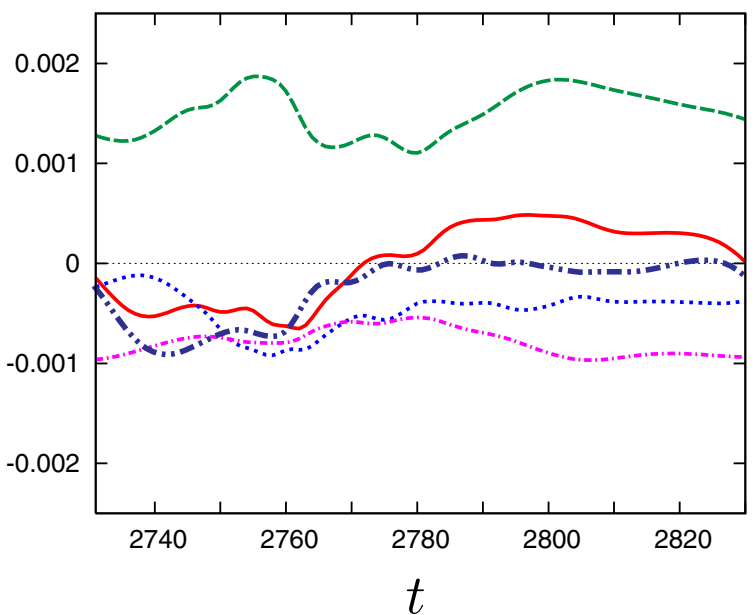

FIG. 17. (Color online) Budget analysis of the evolution equation of the streak mode "energy" Eq. (11). The red (solid) line, the green (dashed) line, and the pink (dashed-dot) line are the same as in Fig. 15. The blue (dot) line is the other nonlinear terms and the navy (dashed double-dotted) line is the nonlinear interaction terms with the meandering mode in Eq. (11).

interaction terms with the meandering mode $\left\langle h\left(\boldsymbol{k}_{s}, z\right)\right\rangle_{z}$ in Eq. (11). The red, green, and pink lines are the same as those in Fig. 15. It is found that the interaction terms with the meandering mode are negative during the phase (i): $\left\langle h\left(\boldsymbol{k}_{s}, z\right)\right\rangle_{z} \simeq-0.001$ (the navy dashed double-dotted line in Fig. 15), and almost zero during the phase (ii): $\left\langle h\left(\boldsymbol{k}_{s}, z\right)\right\rangle_{z} \simeq 0$, while the other nonlinear terms in Eq. (11) do not exhibit a drastic change throughout the cycle (the blue dotted line in Fig. 15).

Finally, in Fig. 18 we show time series of each term of the simplified modal energy equation

$$
\frac{d}{d t}\left\langle\left|\hat{\boldsymbol{u}}\left(\boldsymbol{k}_{s}\right)\right|^{2}\right\rangle_{z}=\left\langle h\left(\boldsymbol{k}_{s}, z\right)\right\rangle_{z}+\left\langle r\left(\boldsymbol{k}_{s}, z\right)\right\rangle_{z},
$$

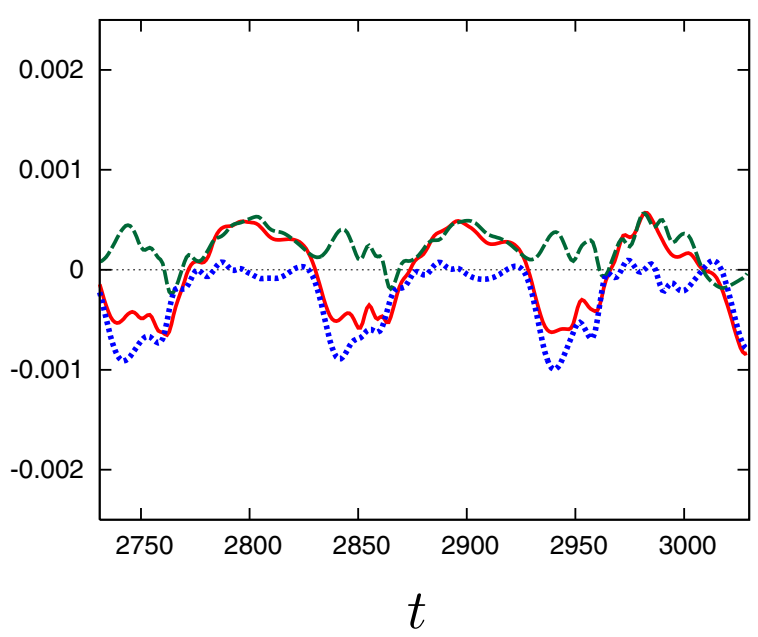

FIG. 18. (Color online) Budget analysis of the evolution equation of the streak mode "energy" Eq. (13) for $2730 \leqslant t \leqslant 3030$ including nearly three regeneration cycles. The red (solid) line is the time derivative term [left-hand side of Eq. (13)], the blue (dot) line is the interaction term between the streak mode and the meandering mode $\left\langle h\left(\boldsymbol{k}_{s}, z\right)\right\rangle_{z}$, and the green (dashed) line is the rest term $\left\langle r\left(\boldsymbol{k}_{s}, z\right)\right\rangle_{z}$. where $\left\langle h\left(\boldsymbol{k}_{s}, z\right)\right\rangle_{z}$ is the interaction term between the streak mode and the meandering mode, and $\left\langle r\left(\boldsymbol{k}_{s}, z\right)\right\rangle_{z}$ denotes the rest term containing the first term and the third and fourth terms on the right-hand side of Eq. (11). The time series include nearly 3 regeneration cycles $(2730 \leqslant t \leqslant 3030)$. The rest term $\left\langle r\left(\boldsymbol{k}_{s}, z\right)\right\rangle_{z}$ is positive and is nearly constant at all times. In contrast, the interaction term between the streak mode and the meandering mode $\left\langle h\left(\boldsymbol{k}_{s}, z\right)\right\rangle_{z}$ is negative at phase (i), and nearly zero at phase (ii).

These observations can be interpreted as follows: when the interaction between the streak mode and the meandering mode is "active," $\left\langle h\left(\boldsymbol{k}_{s}, z\right)\right\rangle_{z}<0$ [phase (i)], the energy leaks from the streak mode, and when the interaction is "inactive," $\left\langle h\left(\boldsymbol{k}_{s}, z\right)\right\rangle_{z} \simeq 0$ [phase (ii)], the energy accumulates in the streak mode. Therefore, we could conclude that the interaction between the streak mode and the meandering mode $\left\langle h\left(\boldsymbol{k}_{s}, z\right)\right\rangle_{z}$ controls the cycle.

\section{CONCLUSION AND DISCUSSION}

In this paper, we considered the characterization of the regeneration cycle in the minimal Couette turbulence with the orbital instability. The orbital instability was studied by using the covariant Lyapunov analysis applied to the full Navier-Stokes equation rather than by using modelings or by phenomenological arguments.

First, the Lyapunov spectrum of the minimal Couette turbulence shows the following results:

The maximum Lyapunov exponent $\lambda_{1}$. The maximum Lyapunov exponent is $\lambda_{1}=0.021$. Interestingly, the value of $\lambda_{1}$ is close to the maximum Floquet exponent $(\mu=0.019)$ of the strong UPO reported by Kawahara [18]. Kawahara and Kida [19] showed that the statistics of the minimal Couette turbulence can be approximated well by the strong UPO. Saiki and Yamada [31,32] studied the statistics on the segments of the chaotic orbits and those of the UPOs numerically. They found a relation that the UPO whose Floquet exponent is close to the Lyapunov exponent gives a good approximation to the statistics of the chaotic attractor (see Fig. 2 in [32]). If the relation holds in our case, we can conclude as follows: the reason why the strong UPO can approximate the statistics of the minimal Couette turbulence is that the Floquet exponent of the UPO is close to the maximum Lyapunov exponent of turbulence. Nikitin [33] studied the maximum Lyapunov exponent of developed wall turbulence in a circular tube and a plane channel. They found that the maximum Lyapunov exponent normalized by the wall time scale was estimated to be a constant value $\left(\lambda_{N}^{+} \approx 0.021\right)$ which is independent of the Reynolds number in the range of $4000 \leqslant \operatorname{Re} \leqslant 10700(140 \leqslant$ $\left.\mathrm{Re}_{\tau} \leqslant 320\right)$ and type of the boundary shape. In the case of the minimal Couette turbulence studied here, the maximum Lyapunov exponent normalized by the wall time scale is $\lambda_{1}^{+} \approx 0.007$ which is about one third of the exponent $\lambda_{N}^{+}$found by Nikitin $\left[\lambda_{1}^{+}=\lambda_{1} t_{\tau} \approx 0.007\right.$, where the wall time scale $t_{\tau}$ is $\left.t_{\tau}=l_{\tau} / u_{\tau}=\operatorname{Re} / \operatorname{Re}_{\tau}^{2}=0.346\left(\operatorname{Re}=400, \operatorname{Re}_{\tau}=34\right)\right]$. It is possible that the large difference in the Reynolds number causes this discrepancy between the exponent of the minimal Couette turbulence and that of the developed wall turbulence. It is expected that the maximum Lyapunov exponent $\lambda_{1}^{+}$ 
(i)
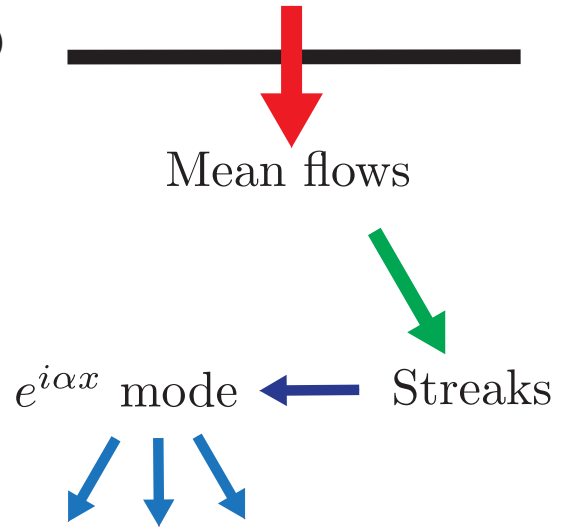

(ii)

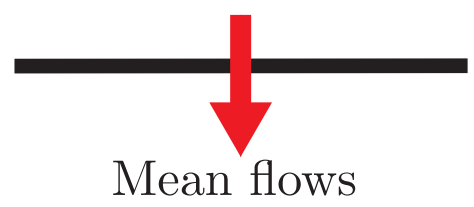

$e^{i \alpha x}$ mode

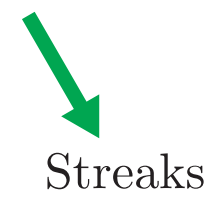

FIG. 19. (Color online) Conceptual diagram of the energy flows in the regeneration cycle for phases (i) and (ii). In the diagrams, "Mean flows" denotes $\boldsymbol{k}=(0,0)$ mode, "Streaks" denotes streak mode $\left(\boldsymbol{k}_{s}=(0,1)\right)$, and " $e^{i \alpha x}$ mode" denotes meandering mode $\left[\boldsymbol{k}_{m}=(1,0)\right]$.

increases with the Reynolds number, and attains to the constant value $\lambda_{N}^{+} \approx 0.021$.

Dimension of the unstable manifold. Dimension of the unstable manifold of the attractor is the number of positive Lyapunov exponents. In the case of the minimal Couette flow, the dimension of the unstable manifold is four. The strong UPO has one positive and a complex conjugate pair of unstable Floquet multipliers [18], which means the dimension of the unstable manifold of the strong UPO is three. While the strong UPO approximates well the statistics of the minimal Couette turbulence, the turbulence attractor possesses another unstable direction which cannot be captured by the strong UPO only.

Dimension of the attractor. Dimension of the turbulence attractor was calculated as $D_{L} \simeq 14.8$ by using the KaplanYorke formula. This low dimensionality may be the reason why the low-dimensional models can reproduce the behaviors like the regeneration cycle. The attractor dimension of the turbulent Poiseuille flow was estimated as $D_{L} \simeq 780$ at the (not so high) Reynolds number $\operatorname{Re}=3200\left(\operatorname{Re}_{\tau}=80\right)$ [34]. Therefore, the attractor dimension of the wall turbulence is considered to increase drastically with the Reynolds number.

Second, we studied the temporal variation of the local Lyapunov exponents of the regeneration cycle. We divided the regeneration cycle into two phases; i.e., phase (i) is the streak breakdown period and phase (ii) is the streak reformation period. We concluded that the flow is unstable only in phase (i), and there is no instability in phase (ii), by observing the local Lyapunov exponents and the finite time growth rate.

We examined the Lyapunov vectors (modes) associated with the positive Lyapunov exponents, which gives more detailed information on the flow instability. We observed the sinuous instability at the initial stage of phase (i), and the streamwise vortices instability at the final stage of phase (i). The local Lyapunov exponent related to the streamwise vortices instability is larger than that of the sinuous instability. Therefore, the streamwise vortices instability is the strongest in magnitude of the local-in-time instability. Phase (ii) may be interpreted as the period in which the orbit far from UPO in phase (i) returns to UPO along its stable manifold, although the confirmation of this interpretation would require a careful study of stable manifolds of UPO not being discussed in this paper.
Finally, we studied what drives phase (ii) and the streak formation, by employing an energy budget analysis. As a result, we found that the energy is injected into the streak mode nearly constantly at all time through the mean flow interaction, not into the meandering mode, and the energy for the reformation of the streaks comes through the mean flow interaction. Besides, we concluded that the streak and meandering mode interaction $\left\langle h\left(\boldsymbol{k}_{s}, z\right)\right\rangle_{z}$ controls the cycle.

To summarize our results, we illustrate the energy flow in a schematic diagram, Fig. 19. The horizontal black lines represent walls. Here we illustrate only the dominant energy flows in the system, and skip to draw the relatively small or nearly constant energy flows such as the energy dissipation. Energy is injected from the walls into the mean flows directly (red arrows). Then, in both the phases, the energy flows (green arrows) into the streak mode ("streaks" in the diagram), not

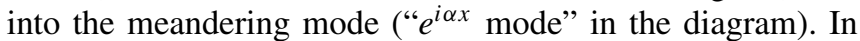
phase (ii) [Fig. 19, panel (ii)], the energy accumulates in the streak mode and its amplitude increases with time (streak formation period). Eventually, the streaks become unstable to the sinuous mode if the amplitude exceeds a certain threshold, which leads to the streak breakdown [phase (i)]. In phase (i), the streak modal energy leaks into the meandering mode (navy arrow) through the interaction between them $\left\langle h\left(\boldsymbol{k}_{s}, z\right)\right\rangle_{z}$. The energy flows into the higher wave number mode (blue arrow) in phase (i). The sinuous instability makes the streaks meander, and then the streamwise vortices localize, which results in the largest local Lyapunov exponent in the cycle. The streamwise vortices grow and break the large scale flow structure into small ones. And then, the streak and the meandering modes interaction $\left\langle h\left(\boldsymbol{k}_{s}, z\right)\right\rangle_{z}$ becomes inactive; then the energy accumulates in the streak mode again, which closes the regeneration cycle.

As a future work, it would be interesting to see if the orbital instabilities found in the minimal Couette turbulence are common characteristics of the regeneration cycle in various minimal wall turbulence, and how the orbital instabilities of the cycle change with increasing Reynolds number or system size. Characterization of the robust layer in the velocity profiles such as viscous, buffer, and logarithmic layer in terms of Lyapunov modes would be also a future problem. As a reference, in the Rayleigh-Bénard convection, Karimi and Paul 
[35] showed statistically that a transition from "boundarydominated" dynamics to "bulk-dominated" dynamics occurs as the system size is increased by employing the Lyapunov mode associated with the largest Lyapunov exponent.

In this paper, we studied the regeneration cycle only. However, as well as the regeneration cycle, the so-called bursting event also occurs infrequently in wall turbulence, and is considered as an important phenomenon [24,36,37]. It is challenging to characterize the bursting event from the standpoint of the orbital instability and clarify the bursting mechanism. For instance, Kobayashi and Yamada [38] studied intermittency in the GOY shell model and they characterized the bursting phenomenon in the GOY shell model with stable and unstable manifold structures via the covariant Lyapunov analysis. It would also be interesting to study the bursting phenomenon in wall turbulence in terms of such manifold structures.

\section{ACKNOWLEDGMENTS}

This work was supported by JSPS KAKENHI Grants No. 24340016, No. 15K13458, No. 22654014, No. 25610139, and No. 26103706 and by Grant-in-Aid for JSPS Fellows No. 24-3995. The numerical calculations were performed on the computer systems of the Institute for Information Management and Communication (IIMC) of Kyoto University and of the Research Institute for Mathematical Sciences, Kyoto University.
[1] F. Ginelli, P. Poggi, A. Turchi, H. Chaté, R. Livi, and A. Politi, Phys. Rev. Lett. 99, 130601 (2007).

[2] H.-L. Yang, K. A. Takeuchi, F. Ginelli, H. Chaté, and G. Radons, Phys. Rev. Lett. 102, 074102 (2009).

[3] P. V. Kuptsov and S. P. Kuznetsov, Phys. Rev. E 80, 016205 (2009).

[4] K. A. Takeuchi, H.-1. Yang, F. Ginelli, G. Radons, and H. Chaté, Phys. Rev. E 84, 046214 (2011).

[5] M. Inubushi, M. U. Kobayashi, S.-i. Takehiro, and M. Yamada, Phys. Rev. E 85, 016331 (2012).

[6] M. Inubushi, M. U. Kobayashi, S. Takehiro, and M. Yamada, Procedia IUTAM 5, 244 (2012).

[7] H.-L. Yang and G. Radons, Phys. Rev. Lett. 108, 154101 (2012).

[8] P. V. Kuptsov and U. Parlitz, J. Nonlinear Sci. 22, 727 (2012).

[9] F. Ginelli, H. Chate, R. Livi, and A. Politi, J. Phys. A: Math. Theor. 46, 254005 (2013).

[10] G. Froyland, T. Huls, G. P. Morriss, and T. M. Watson, Physica D 247, 18 (2013).

[11] J. Jimenez and P. Moin, J. Fluid Mech. 225, 213 (1991).

[12] J. M. Hamilton, J. Kim, and F. Waleffe, J. Fluid Mech. 287, 317 (1995).

[13] R. L. Panton, Prog. Aerospace Sci. 37, 341 (2001).

[14] T. Duriez, J.-L. Aider, and J. E. Wesfreid, Phys. Rev. Lett. 103, 144502 (2009).

[15] F. Waleffe, Phys. Fluids 9, 883 (1997).

[16] W. Schoppa and F. Hussain, J. Fluid Mech. 453, 57 (2002).

[17] G. Kawahara, J. Jimenez, M. Uhlmann, and A. Pinelli, J. Fluid Mech. 483, 315 (2003).

[18] G. Kawahara, Fluid Dyn. Res. 41, 064001 (2009).
[19] G. Kawahara and G. Kida, J. Fluid Mech. 449, 291 (2001).

[20] F. Waleffe, J. Fluid Mech. 435, 93 (2001).

[21] D. Viswanath, J. Fluid Mech. 580, 339 (2007).

[22] J. F. Gibson, J. Halcrow, and P. Cvitanovic, J. Fluid Mech. 611, 107 (2008).

[23] J. Halcrow, J. F. Gibson, P. Cvitanovic, and D. Viswanath, J. Fluid Mech. 621, 365 (2009).

[24] L. van Veen and G. Kawahara, Phys. Rev. Lett. 107, 114501 (2011).

[25] G. Kawahara, Annu. Rev. Fluid Mech. 44, 203 (2012).

[26] J. Philip and P. Manneville, Phys. Rev. E 83, 036308 (2011).

[27] K. Ishioka, ispack-0.95, http://www.gfd-dennou.org/arch/ ispack, GFD Dennou Club.

[28] S. Takehiro, M. Odaka, K. Ishioka, M. Ishiwatari, and Y. Hayashi, SPMODEL: A series of Hierarchical Spectral Models for Geophysical Fluid Dynamics, Nagare Multimedia, 2006. Available online at http://www.nagare.or.jp/mm/2006/spmodel.

[29] See http://www.gfd-dennou.org/library/ruby.

[30] I. Shimada and T. Nagashima, Prog. Theor. Phys. 61, 1605 (1979).

[31] Y. Saiki and M. Yamada, Phys. Rev. E 79, 015201 (2009).

[32] Y. Saiki and M. Yamada, RIMS Kokyuroku 1768, 143 (2011).

[33] N. V. Nikitin, Fluid Dyn. 44, 652 (2009).

[34] L. Keefe, P. Moin, and J. Kim, J. Fluid Mech. 242, 1 (1992).

[35] A. Karimi and M. R. Paul, Phys. Rev. E 85, 046201 (2012).

[36] T. Itano and S. Toh, J. Phys. Soc. Jpn. 70, 703 (2001).

[37] J. Jiménez, G. Kawahara, M. P. Simens, M. Nagata, and M. Shiba, Phys. Fluids 17, 015105 (2005).

[38] M. U. Kobayashi and M. Yamada, J. Phys. A: Math. Theor. 46, 254008 (2013) 